\title{
Difference in Bose-Einstein condensation of conserved and unconserved particles
}

\author{
V.I. Yukalov \\ Bogolubov Laboratory of Theoretical Physics, \\ Joint Institute for Nuclear Research, Dubna 141980, Russia
}

\begin{abstract}
Keywords: Bose-Einstein condensation, trapped atoms, optical lattices, conserved and unconserved particles, quasiparticles, vibrating optical lattices, double-well lattices, condensation of collective excitations, phonons, magnons, Schwinger bosons, slave bosons, dimer lattices, condensation of singletons and triplons, strongly nonequilibrium condensates
\end{abstract}

\begin{abstract}
The peculiarities in the Bose-Einstein condensation of particles and quasiparticles are discussed. The difference between the condensation of conserved and unconserved particles is analyzed. A classification of quasiparticles is given. The emphasis is made on the ability of particles and quasiparticles to condense. Illustrations include: general Bose-condensed atomic systems, such as ensembles of trapped atoms, Bose gases with conserved and unconserved number of atoms, vibrating atoms in double-well lattices, Holstein-Primakoff magnons, Schwinger bosons, slave bosons, and the condensation of singletons and triplons. The basic difference is that the system of particles, whose total number is conserved, can form equilibrium as well as nonequilibrium condensates, while unconserved particles can condense only in a nonequilibrium system subject to external pumping supporting the density of these particles sufficient for their condensation. The examples of such a nonequilibrium condensation of unconserved particles are the Bose-Einstein condensation of excitons, polaritons, and photons. Elementary collective excitations, such as bogolons and phonons, being self-consistently defined, do not condense. Magnons cannot condense in equilibrium systems. Controversies, existing in literature with regard to the Bose-Einstein condensation of some quasiparticles, are explained. Pushing a system out of equilibrium may favor the condensation of unconserved quasiparticles, but suppresses the condensate fraction of conserved particles.
\end{abstract}




\section{Introduction}

Bose-Einstein condensation has been a topic of intensive studies, both experimental and theoretical. There exists numerous literature on the problem, which can be found in the books [1-4] and review articles [5-18]. A variety of experiments have been performed with cold trapped atoms. In addition to atoms, whose total average number can be well controlled, hence, is conserved, one often considers Bose-Einstein condensation of unconserved particles, e.g., corresponding to excited bound states or collective excitations. The known examples are the Bose-Einstein condensation of excitons [19-23], polaritons [24-27], and, recently, photons [28].

Sometimes controversy arises, accompanying the discussion on the feasibility of realizing the Bose-Einstein condensation of unconserved particles. A very illustrative case of such a controversy is the discussion on the possibility of magnon condensation. In several works (e.g. [29-32]), the authors claimed the existence of magnon condensation in some equilibrium magnetic materials. Because of the known mapping $[33,34]$ of spin systems to Bose gas, the existence of magnon condensation would mean the occurrence of Bose condensation for the collective excitations (bogolons) of Bose systems. Is it feasible that bogolons would condense, may be in a nonequilibrium Bose gas [35] in an external alternating potential? The problems of whether bogolons and magnons could condense are closely related. Mills [36] stressed that magnon condensation in equilibrium systems is impossible. Why this is impossible and could magnons condense in nonequilibrium systems? More generally, could elementary collective excitations become condensed and, if so, when?

Understanding the necessary conditions, when particles and quasiparticles could form Bose-Einstein condensates, is a general problem that has become of great importance due to high current interest to Bose-condensed systems [1-18]. In the present paper, a careful analysis is given of such necessary conditions of condensation. The consideration is illustrated by the cases of the most common types of quasiparticles whose classification is also given. The suggested analysis resolves some controversies arising with regard to the possibility of Bose-Einstein condensation of quasiparticles.

\section{$2 \quad$ Particles versus quasiparticles}

Traditionally, one distinguishes elementary particles and quasiparticles. The distinction, however, can differ depending on the considered physical applications.

In particle physics, an elementary particle, or fundamental particle, is a particle not known to have substructure; that is, it is not known to be made up of smaller particles. If an elementary particle truly has no substructure, then it is one of the basic building blocks of the universe from which all other particles are made. In the Standard Model, the quarks, leptons, and gauge bosons are elementary particles [37].

In statistical physics, one calls an elementary particle that whose structure is either absent or of no importance for the considered application. So, hadrons (mesons and baryons, such as the proton and neutron) and even whole atoms and molecules may be regarded as elementary particles, provided that their structure, if it exists, can be neglected for the studied phenomena.

The notion of quasiparticles is not well defined. One often calls quasiparticles the dressed 
particles whose properties are changed by the surrounding medium. For example, as an electron travels through a semiconductor, its motion is disturbed in a complex way by its interactions with all of the other electrons and nuclei. However it approximately behaves like an electron with a different mass traveling unperturbed through free space. This "electron" with a different mass is called an electron quasiparticle or Landau quasiparticle. Various single-particle and collective excitations are also termed quasiparticles. To make the consideration better defined, we give below a classification of different types of quasiparticles.

\subsection{Dressed particles}

Dressed particles are those that can be treated as elementary, but with the properties that are changed by surrounding, making them different from their properties in vacuum. Examples are: electron in a solid, electron or ion in plasma, proton or neutron inside a nucleus, polaron (electron with polarization of surrounding ions of a crystalline lattice), polariton (photon in a medium). Sometimes composite particles can mathematically be treated as elementary, such as atoms and molecules. A stable bipolaron (bound pair of two polarons) can be considered as one Bose particle. Strictly speaking, in quantum field theory, all particles interact with vacuum, being in that sense all dressed. The dressed particles can be bosons or fermions depending on their spin.

\subsection{Particle excitations}

Some quasiparticles behave as dressed particles, but differ from them by the necessity of supplying energy to the system for their creation. In that sense, such particle excitations are not stable and decay in an equilibrium system. For instance, a hole in the valence band of a semiconductor is a lack of an electron that has been excited to the conductance band. A hole is a fermion quasiparticle. Another example is an exciton in a semiconductor, that is a bound state of an electron and a hole, requiring for its creation photon excitation. Excitons can be treated as bosons. The number of excitations is not conserved, depending on the intensity of the applied external pumping.

\subsection{Collective excitations}

Collective excitations describe quantized fluctuations of an order parameter. Examples are: phonon (quant of mass density fluctuations), plasmon (quant of charge density fluctuations), magnon (quant of spin fluctuations), bogolon (quant of density fluctuations in a Bose-condensed system). Such collective excitations are usually described by linear equations characterizing small deviations from a stationary state, because of which they are termed elementary collective excitations. All these collective excitations are bosons. They are not conserved.

\subsection{Nonlinear waves}

Another type of excitations can also be formed by the system as a whole, being in that sense also collective, but, contrary to the elementary collective excitations, being described 
by nonlinear equations corresponding to large deviations from a stationary state. In classical and quasiclassical picture such excitations are named nonlinear waves. As examples, we can mention solitons [38,39], bions (two bound solitons) [40,41], triple solitons (three bound solitons) [42], quantum vortices [17], and topological coherent modes [43-54]. Being quantized, such nonlinear excitations can be interpreted as quasiparticles. Usually they are not stable, except some one-dimensional solitons, arise only in nonequilibrium systems, and are not conserved.

\subsection{Auxiliary quasiparticles}

In quantum theory, particles and quasiparticles are represented by field operators of creation and annihilation. The operators can be transformed resulting in the appearance of new operators that can correspond to other physical quasiparticles. Thus, the field operator of uncondensed particles in a Bose-condensed system can be decomposed into the linear combination of operators corresponding to collective excitations, bogolons. But it is not necessary that any operator transformation would result in new operators corresponding to some physical particles or quasiparticles. A transformation can be just a mathematical formula, convenient for calculations, but not necessarily related to new physical objects. Nevertheless, it is customary to associate each field operator, occurring in operator transformations, with a quasiparticle, though this can be merely a fictitious particle being related to no real physical object. Such auxiliary fields and quasiparticles occur, e.g., in the HubbardStratonovich transformation [55]. Other examples will be given below. Auxiliary particles can be either bosons or fermions, or even anyons, having intermediate fractional statistics $[56]$.

\section{Condensation of cold atoms}

There are two ways of treating Bose-Einstein condensation of particles whose total number is fixed. It is possible to consider a finite system of $N$ particles in volume $V$. In a finite system, strictly defined phase transitions are absent. Following a kind of the Penrose-Onsager scheme [57], one needs to find the largest eigenvalue of the single-particle density matrix, which represents the number of condensed particles $N_{0}$. If the value $n_{0} \equiv N_{0} / N$ in thermodynamic limit remains finite, one says that Bose-Einstein condensation takes place. In that case, the order index of the single-particle density matrix becomes unity [58-60]. This way is extremely complicated and, for realistic models, can be realized only by means of powerful computers.

Another way takes into account that, though strictly defined phase transitions, including Bose-Einstein condensation, occur only in thermodynamic limit, but, if the number of particles in the considered system is sufficiently large, such that $N \gg 1$, then one can use the relations typical of infinite systems, where phase transitions are well defined. This essentially simplifies the consideration. In thermodynamic limit, Bose condensation is accompanied by spontaneous breaking of global gauge symmetry. Moreover, the symmetry breaking is necessary and sufficient for Bose-Einstein condensation [11]. In a finite system, the symmetry breaking is asymptotic, in the sense that it is approximate for finite $N$, and becomes exact for $N \rightarrow \infty$. For a large number of particles $N \gg 1$, the symmetry can be considered as broken. Then it is reasonable to take into account the symmetry breaking explicitly, which 
makes the theory more transparent and calculations treatable.

In the case of Bose condensation, the most convenient method of symmetry breaking is by means of the Bogolubov shift $[61,62]$, when the Bose field operator is represented as the sum

$$
\hat{\psi}(\mathbf{r}, t)=\eta(\mathbf{r}, t)+\psi_{1}(\mathbf{r}, t)
$$

in which the first term is the condensate wave function and the second term is the field operator of uncondensed atoms. The latter two quantities are orthogonal to each other,

$$
\int \eta^{*}(\mathbf{r}, t) \psi_{1}(\mathbf{r}, t) d \mathbf{r}=0
$$

Uncondensed atoms are considered as normal, such that the statistical average of the operator of uncondensed atoms is zero:

$$
\left\langle\psi_{1}(\mathbf{r}, t)\right\rangle=0
$$

Then the average of the total field operator (1),

$$
\langle\hat{\psi}(\mathbf{r}, t)\rangle=\eta(\mathbf{r}, t)
$$

gives the condensate wave function that can be treated as the order parameter. Its modulus squared yields the condensate density

$$
\rho_{0}(\mathbf{r}, t)=|\eta(\mathbf{r}, t)|^{2}
$$

which is normalized to the number of condensed atoms

$$
\int|\eta(\mathbf{r}, t)|^{2} d \mathbf{r}=N_{0}(t) .
$$

Another normalization condition gives the number of uncondensed atoms

$$
\left\langle\hat{N}_{1}(t)\right\rangle=N_{1}(t)
$$

that is the average of the number operator

$$
\hat{N}_{1}(t) \equiv \int \psi_{1}^{\dagger}(\mathbf{r}, t) \psi_{1}(\mathbf{r}, t) d \mathbf{r}
$$

The sum of the numbers of condensed, (6), and uncondensed, (7), atoms gives the total number of atoms

$$
N_{0}(t)+N_{1}(t)=N
$$

Generally, the total number of atoms could depend on time, when there is the input of atoms or their loss. But in any case, this number is fixed at each moment of time by the given external conditions and cannot be created inside the system.

Equation (3) can be rewritten in the standard form of a statistical condition by introducing the operator

$$
\hat{\Lambda}(t) \equiv \int\left[\lambda(\mathbf{r}, t) \psi_{1}^{\dagger}(\mathbf{r}, t)+\lambda^{*}(\mathbf{r}, t) \psi_{1}(\mathbf{r}, t)\right] d \mathbf{r}
$$


Then Eq. (3) is equivalent to the condition

$$
\langle\hat{\Lambda}(t)\rangle=0
$$

The above formulas are defined for an arbitrary system, equilibrium or nonequilibrium, provided that the condensate does exist, that is, when the condition of condensate existence

$$
\lim _{N \rightarrow \infty} \frac{N_{0}(t)}{N}>0
$$

is valid. The system as such can be finite, but the thermodynamic limit in Eq. (12) serves as the check for the accurate definition of the condensate existence. This condition imposes constraints on the behavior of the distribution of uncondensed atoms and the spectrum of collective excitations $[16,18]$ for an equilibrium system. The distribution of atoms $n_{k}$ over the quantum multi-indices $k$ is to be such that, if the condensation is into the state labelled by $k_{0}$, then

$$
n_{k} \rightarrow \infty \quad\left(k \rightarrow k_{0}\right) .
$$

This is equivalent to the requirement that the spectrum $\varepsilon_{k}$ be gapless:

$$
\varepsilon_{k} \rightarrow 0 \quad\left(k \rightarrow k_{0}\right)
$$

Another important point is that the cases, when the condensate exists or does not exist require different mathematics [63]. When the condensate exists, hence condition (12) holds true, then the action of all operators is defined on the Fock space $\mathcal{F}\left(\psi_{1}\right)$ generated by the field operator $\psi_{1}$. But when there is no condensate, so that the condensate function $\eta(\mathbf{r}, t)$ and the condensate density (5) are zero, then the theory is defined on the Fock space $\mathcal{F}(\psi)$ generated by the field operator $\psi$, without any shift. These two spaces are orthogonal to each other [63]. It is incorrect to work in the space $\mathcal{F}(\psi)$ and then make the Bogolubov shift (1) passing to the space $\mathcal{F}\left(\psi_{1}\right)$. This is the standard mistake. As soon as there happens Bose-Einstein condensation, one has to work in the space $\mathcal{F}\left(\psi_{1}\right)$.

Let $\hat{H}\left[\eta, \psi_{1}\right]$ be the energy Hamiltonian for the system. Because of the existence of three statistical conditions (6), (7), and (11), the grand Hamiltonian must contain three additional terms:

$$
H=\hat{H}\left[\eta, \psi_{1}\right]-\mu_{0} N_{0}(t)-\mu_{1} \hat{N}_{1}(t)-\hat{\Lambda}(t) .
$$

Here $\mu_{0}$ and $\mu_{1}$ are the Lagrange multipliers guaranteeing the normalizations (6) and (7). And $\lambda(\mathbf{r}, t)$ is the Lagrange multiplier cancelling the linear in $\psi_{1}(\mathbf{r}, t)$ terms in order to satisfy condition (11). The grand Hamiltonian (15) allows one to correctly define a representative statistical ensemble uniquely describing the Bose-condensed system [63-67].

The equations of motion can be represented by the variational derivatives of the grand Hamiltonian over $\eta$ and $\psi_{1}$, which is equivalent to the Heisenberg equations of motion [18,68]. Thus, the equation for the condensate wave function reads as

$$
i \frac{\partial}{\partial t} \eta(\mathbf{r}, t)=\left\langle\frac{\delta H}{\delta \eta^{*}(\mathbf{r}, t)}\right\rangle .
$$

In the case of an equilibrium system, this reduces to the equation

$$
\left\langle\frac{\delta H}{\delta \eta^{*}(\mathbf{r})}\right\rangle=0 \text {. }
$$


And, if the equilibrium system is uniform, the previous equation becomes

$$
\left\langle\frac{\partial H}{\partial N_{0}}\right\rangle=0 .
$$

Evolution equations, together with the related initial conditions, define the functions $N_{0}\left(t, \mu_{0}, \mu_{1}\right)$ and $N_{1}\left(t, \mu_{0}, \mu_{1}\right)$. Because of normalization (9), the multipliers $\mu_{0}$ and $\mu_{1}$ are connected with each other and, generally, can depend on time. The condition of the gapless spectrum, for a nonequilibrium system, reads as

$$
\varepsilon_{k}(t) \rightarrow 0 \quad\left(k \rightarrow k_{0}\right) .
$$

These equations define the multipliers $\mu_{0}(t, N)$ and $\mu_{1}(t, N)$.

The time-dependent solutions should tend to the corresponding stable stationary solutions $N_{0}=N_{0}\left(\mu_{0}, \mu_{1}\right)$ and $N_{1}=N_{1}\left(\mu_{0}, \mu_{1}\right)$ that, according to sum (9), satisfy the normalization $N_{0}+N_{1}=N$. The condition of condensate existence, in the form of the gapless-spectrum requirement (14), gives $\mu_{1}=\mu_{1}\left(\mu_{0}, N\right)$ by the Hugenholtz-Pines relation [69]. These four equations uniquely define all four quantities: $N_{0}, N_{1}, \mu_{0}, \mu_{1}$ through the parameters of the system in the stationary state.

If one would assume the equivalence of the chemical potentials $\mu_{0}$ and $\mu_{1}$, this would overdefine the equations and would lead to inconsistences, as has been shown by Hohenberg and Martin [70]. Such inconsistences would lead either to the appearance of a gap in the spectrum or to the invalidity of the equation (16) for the condensate wave function. In turn, thermodynamic relations would break down and the poles of the single-particle and twoparticle Green functions would be different, contradicting the results of Bogolubov [62] and Gavoret and Noziére [71], according to which the poles of these Green functions coincide, when the global gauge symmetry is broken.

The Bogolubov canonical transformation

$$
\psi_{1}(\mathbf{r}, t)=\sum_{k}\left[u_{k}(\mathbf{r}, t) b_{k}(t)+v_{k}^{*}(\mathbf{r}, t) b_{k}^{\dagger}(t)\right]
$$

introduces the Bose operators $b_{k}(t)$ of collective excitations, bogolons, where $k$ is a quantum multi-index. This transformation makes it possible to diagonalize the grand Hamiltonian in the Hartree-Fock-Bogolubov approximation. The coefficient functions $u_{k}$ and $v_{k}$ are found from this diagonalization which yields the Bogolubov equations that also give the Bogolubov spectrum $\varepsilon_{k}$. The bogolon number is not conserved and bogolons cannot condense. This is evident from relation (20). Really, if the bogolons would condense, resulting in nonzero $\left\langle b_{k}(t)\right\rangle$, then the average $\left\langle\psi_{1}(\mathbf{r}, t)\right\rangle$ would also become nonzero, which contradicts the basic definition (3).

Thus, atoms, whose total number $N$ is given, can form an equilibrium, as well as nonequilibrium, Bose-Einstein condensate. But the collective excitations, bogolons, whose number is not conserved, cannot be condensed. If in a nonequilibrium system one would notice the tendency of bogolons to condense, this would simply imply the necessity of redefining the condensate function of atoms in such a way that to preserve the principal definition (4), hence, avoiding the bogolon condensation. The same concerns other collective excitations that could arise in Bose-condensed systems, even if these excitations would have single-particle form [72]. The main point is that the collective excitations correspond to 
unconserved entities, so that they cannot condense. As is clear from the present section, bogolons, that are elementary collective excitations in a Bose-condensed system, by definition cannot condense whether in equilibrium or in any nonequilibrium case.

\section{Conserved versus unconserved particles}

As an explicit illustration demonstrating the basic difference between conserved and unconserved quasiparticles and the impossibility of equilibrium condensation for unconserved quasiparticles, let us consider boson quasiparticles with the effective spectrum

$$
\omega_{k}=A k^{n}
$$

where $A$ and $n$ are positive parameters and $k$ is the modulus of a $d$-dimensional wave vector. This type of spectrum occurs in some problems of solid-state physics and some cosmological problems, where the matter with such a spectrum is named absolute stiff matter [73-76]. Quasiparticles are assumed to be in a large box of volume $V$, so that the sum over $d$ dimensional momenta can be replaced by the integral

$$
\sum_{k} \longrightarrow V \int \frac{d \mathbf{k}}{(2 \pi)^{d}} \longrightarrow V \int_{0}^{\infty} \frac{2 k^{d-1} d k}{(4 \pi)^{d / 2} \Gamma(d / 2)}
$$

The uniform density is denoted by $\rho \equiv N / V$.

\subsection{Equilibrium condensation of conserved quasiparticles}

Here we consider conserved quasiparticles, whose total number $N$ is fixed, therefore the density is constant, $\rho=$ const. The critical temperature, where the condensation starts, is found in the usual way [18], yielding

$$
T_{c}=A\left[\frac{(4 \pi)^{d / 2} \Gamma(d / 2) n \rho}{2 \Gamma(d / n) \zeta(d / n)}\right]^{n / d} .
$$

Taking into account that the gamma-function $\Gamma(x)>0$ for $x>0$ and the Riemann zetafunction $\zeta(x)<0$ in the interval $0<x<1$ tells us that there is no condensation for $d<n$. When $d=n$, then $T_{c}=0$. And $T_{c}$ is positive only for $d>n$. In the latter case, for the condensate fraction below $T_{c}$, one has

$$
n_{0} \equiv \frac{N_{0}}{N}=1-\left(\frac{T}{T_{c}}\right)^{d / n} .
$$

However, the formal existence of the critical temperature does not yet mean that the condensate can really occur. In order to exist, it must represent a stable statistical system. For this purpose, the system compressibility is to be finite, which is equivalent to the finiteness

of the stability ratio

$$
0 \leq \frac{\operatorname{var}(\hat{N})}{N}<\infty
$$


for all $N$, including thermodynamic limit $[7,16,18,77]$. Here

$$
\operatorname{var}(\hat{N}) \equiv\left\langle\hat{N}^{2}\right\rangle-\langle\hat{N}\rangle^{2}, \quad \hat{N} \equiv N_{0}+\hat{N}_{1}
$$

Calculating ratio (24), we use the fact that

$$
\operatorname{var}(\hat{N})=\operatorname{var}\left(\hat{N}_{1}\right)
$$

Then, for $n<d / 2$, we get

$$
\frac{\operatorname{var}(\hat{N})}{N}=\frac{\zeta(d / n-1)}{\zeta(d / n)}\left(\frac{T}{T_{c}}\right)^{d / n} \quad(d>2 n) .
$$

In the interval $d / 2<n<d$, similarly to Ref. [78], we employ the generalized Bose functions, in whose definition the integrals are limited from below by the minimal wave number $k_{\min }=$ $(\rho / N)^{1 / d}$. This gives

$$
\frac{\operatorname{var}(\hat{N})}{N} \approx \frac{2(d-n) N^{(2 n-d) / d}}{(4 \pi)^{d / 2} n(2 n-d) \Gamma(d / 2)}\left(\frac{T}{A \rho^{n / d}}\right)^{2} \quad(n<d<2 n) .
$$

In particular cases, remembering that $T_{c}>0$ exists only for $d>n$, we have the following. When $d=3$ and $n=2$, the critical temperature (22) is

$$
T_{c}=4 \pi A\left[\frac{\rho}{\zeta(3 / 2)}\right]^{2 / 3} \quad(d=3, n=2) .
$$

But ratio (26) diverges for large $N$,

$$
\frac{\operatorname{var}(\hat{N})}{N} \approx\left(\frac{T}{2 \pi A \rho^{2 / 3}}\right)^{2} N^{1 / 3} \quad(d=3, n=2),
$$

hence, the stability condition (24) is not valid.

When $d=2$ and $n=1$, the critical temperature is

$$
T_{c}=2 A \sqrt{\frac{3 \rho}{\pi}} \quad(d=2, n=1) .
$$

But the stability ratio is strongly divergent, hence the system is unstable.

When $d=3$ and $n=1$, the critical temperature (22) becomes

$$
T_{c}=A\left[\frac{\pi^{2} \rho}{\zeta(3)}\right]^{1 / 3} \quad(d=3, n=1),
$$

where we have used the value $\zeta(2)=\pi^{2} / 6$. The stability ratio (25) is finite:

$$
\frac{\operatorname{var}(\hat{N})}{N}=\frac{T^{3}}{6 \rho A^{3}} \quad(d=3, n=1),
$$

telling us that the system is stable.

In this way, the uniform $d$-dimensional Bose gas of conserved quasiparticles with spectrum (21) can be Bose-condensed, provided that $d>2 n$. 


\subsection{No equilibrium condensation of unconserved quasiparticles}

If the total number of quasiparticles is not fixed, but they can be created, then, when decreasing temperature, no condensation occurs, but instead, the number of quasiparticles diminishes with temperature, which implies their decreasing density

$$
\rho=\frac{2 \Gamma(d / n) \zeta(d / n)}{(4 \pi)^{d / 2} n \Gamma(d / 2)}\left(\frac{T}{A}\right)^{d / n} .
$$

This density is positive for $d>n$. In the particular cases, corresponding to those studied above, we have

$$
\begin{gathered}
\rho=\zeta\left(\frac{3}{2}\right)\left(\frac{T}{4 \pi A}\right)^{3 / 2} \quad(d=3, n=2), \\
\rho=\frac{\pi}{3}\left(\frac{T}{2 A}\right)^{2} \quad(d=2, n=1), \\
\rho=\frac{\zeta(3)}{\pi^{2}}\left(\frac{T}{A}\right)^{3} \quad(d=3, n=1) .
\end{gathered}
$$

In all the cases, the density of unconserved quasiparticles diminishes with temperature, tending to zero. But there is no condensation.

The similar situation happens in the case of equilibrium photons. In equilibrium, at lowering temperature, photons disappear instead of occupying the cavity ground state. But under an external pumping supporting photon total number inside an optical microcavity, photons can condense [28]. Such a photon condensation, creating an ensemble of coherent photons is, actually, analogous to the functioning of lasers.

When the lifetime of created quasiparticles is sufficiently long, a quasiequilibrium condensation of unconserved particles in a quasi-stationary state can be realized, as in the case of excitons [19-23,79]. But in absolute equilibrium, unconserved quasiparticles cannot condense.

\section{$5 \quad$ Vibrating optical lattices}

A very common quasiparticle, representing collective excitations, is phonon, characterizing density fluctuations caused by vibration of atoms in a lattice. In addition to the standard solid-state physics, different lattices can be created by means of laser beams forming standing waves where cold atoms are caught $[15,16,80,81]$. Phonons are unconserved quasiparticles. Hence, we should expect that they cannot condense in equilibrium. However, in some cases one talks on condensation of phonons. Here the analysis is given explaining that the phonon condensation in equilibrium systems does not occur. The formal appearance of phonon condensation may happen in nonequilibrium situations, such as phase transitions. But defining phonons in a self-consistent way makes it possible to avoid phonon condensation in any system, equilibrium or not. 


\subsection{Double-well optical lattices}

For generality, let us consider the case of atoms that can happen in two states of a lattice. A straightforward example of such two-state atoms are the atoms in double-well lattices [82-92] and different double-well potentials [93-95]. Another case could correspond to twoband lattices [96]. The usual single-band lattice can be considered as a particular case of a two-band lattice.

The derivation of the lattice model starts with the standard Hamiltonian

$$
\begin{gathered}
\hat{H}=\int \psi^{\dagger}(\mathbf{r}) H_{1}(\mathbf{r}) \psi(\mathbf{r}) d \mathbf{r}+ \\
+\frac{1}{2} \int \psi^{\dagger}(\mathbf{r}) \psi^{\dagger}\left(\mathbf{r}^{\prime}\right) \Phi\left(\mathbf{r}-\mathbf{r}^{\prime}\right) \psi\left(\mathbf{r}^{\prime}\right) \psi(\mathbf{r}) d \mathbf{r} d \mathbf{r}^{\prime},
\end{gathered}
$$

where $\Phi(\mathbf{r})$ is an atomic interaction potential and the single-atom Hamiltonian is

$$
H_{1}(\mathbf{r})=-\frac{\nabla^{2}}{2 m}+V_{l a t}(\mathbf{r})+U_{e x t}(\mathbf{r}, t) .
$$

In the latter, $V_{\text {lat }}(\mathbf{r})$ is a lattice potential and $U_{\text {ext }}(\mathbf{r}, t)$ is an external potential, such as the trapping potential. Generally, the external potential can be time-dependent.

Atoms, located at the spatial points $\mathbf{r}_{j}$, are enumerated by the index $j=1,2, \ldots, N$. Keeping in mind an insulating lattice, we can describe atomic states by the localized orbitals given by the Schrödinger equation

$$
\left[-\frac{\nabla^{2}}{2 m}+V_{l a t}(\mathbf{r})\right] \psi_{n}\left(\mathbf{r}-\mathbf{r}_{j}\right)=E_{n j} \psi_{n}\left(\mathbf{r}-\mathbf{r}_{j}\right) .
$$

The orbitals form an orthonormal basis with the property

$$
\int \psi_{m}^{*}\left(\mathbf{r}-\mathbf{r}_{i}\right) \psi_{n}\left(\mathbf{r}-\mathbf{r}_{j}\right) d \mathbf{r}=\delta_{m n} \delta_{i j} .
$$

The atomic field operator can be expanded over the orbital basis,

$$
\psi(\mathbf{r})=\sum_{n j} c_{n j} \psi_{n}\left(\mathbf{r}-\mathbf{r}_{j}\right) .
$$

We assume that at each location $\mathbf{r}_{j}$ there exists just one atom, which imposes the unipolarity condition

$$
\sum_{n} c_{n j}^{\dagger} c_{n j}=1, \quad c_{m j} c_{n j}=0 .
$$

The matrix element

$$
E_{i j}^{m n}=\int \psi_{m}^{*}\left(\mathbf{r}-\mathbf{r}_{i}\right) H_{1}(\mathbf{r}) \psi_{n}\left(\mathbf{r}-\mathbf{r}_{j}\right) d \mathbf{r}
$$

of the single-atom Hamiltonian (37) is the sum

$$
E_{i j}^{m n}=K_{i j}^{m n}+V_{i j}^{m n}+U_{i j}^{m n}
$$


of the terms

$$
\begin{aligned}
K_{i j}^{m n} & \equiv \int \psi_{m}^{*}\left(\mathbf{r}-\mathbf{r}_{i}\right)\left(-\frac{\nabla^{2}}{2 m}\right) \psi_{n}\left(\mathbf{r}-\mathbf{r}_{j}\right) d \mathbf{r} \\
V_{i j}^{m n} & \equiv \int \psi_{m}^{*}\left(\mathbf{r}-\mathbf{r}_{i}\right) V_{l a t}(\mathbf{r}) \psi_{n}\left(\mathbf{r}-\mathbf{r}_{j}\right) d \mathbf{r} \\
U_{i j}^{m n} & \equiv \int \psi_{m}^{*}\left(\mathbf{r}-\mathbf{r}_{i}\right) U_{e x t}(\mathbf{r}, t) \psi_{n}\left(\mathbf{r}-\mathbf{r}_{j}\right) d \mathbf{r}
\end{aligned}
$$

And the eigenvalues of Eq. (38) are

$$
E_{n j}=K_{j j}^{n n}+V_{j j}^{n n}
$$

Then the matrix elements (42) can be written as

$$
E_{i j}^{m n}=\delta_{m n} \delta_{i j} E_{n j}+U_{i j}^{m n}
$$

We assume that only two lowest bands are occupied, so that $n=1,2$. Then it is possible to introduce the pseudospin operators

$$
\begin{gathered}
S_{j}^{x}=\frac{1}{2}\left(c_{1 j}^{\dagger} c_{1 j}-c_{2 j}^{\dagger} c_{2 j}\right), \quad S_{j}^{y}=\frac{i}{2}\left(c_{1 j}^{\dagger} c_{2 j}-c_{2 j}^{\dagger} c_{1 j}\right), \\
S_{j}^{z}=\frac{1}{2}\left(c_{1 j}^{\dagger} c_{2 j}+c_{2 j}^{\dagger} c_{1 j}\right),
\end{gathered}
$$

which gives the atomic operators

$$
\begin{array}{cl}
c_{1 j}^{\dagger} c_{1 j}=\frac{1}{2}+S_{j}^{x}, & c_{2 j}^{\dagger} c_{2 j}=\frac{1}{2}-S_{j}^{x}, \\
c_{1 j}^{\dagger} c_{2 j}=S_{j}^{z}-i S_{j}^{y}, & c_{2 j}^{\dagger} c_{1 j}=S_{j}^{z}+i S_{j}^{y} .
\end{array}
$$

The pseudospin operators satisfy the standard spin algebra. And the atomic operators can satisfy either boson or fermion commutation relations.

To better understand the physical meaning of the pseudospin operators (45), we can introduce the left and right location operators

$$
c_{j L}=\frac{1}{\sqrt{2}}\left(c_{1 j}+c_{2 j}\right), \quad c_{j R}=\frac{1}{\sqrt{2}}\left(c_{1 j}-c_{2 j}\right)
$$

describing the location of atoms in the left or right wells of a double well that is centered at the lattice point $\mathbf{r}_{j}$. Then the pseudospin operators (45) become

$$
\begin{gathered}
S_{j}^{x}=\frac{1}{2}\left(c_{j L}^{\dagger} c_{j R}+c_{j R}^{\dagger} c_{j L}\right), \quad S_{j}^{y}=-\frac{i}{2}\left(c_{j L}^{\dagger} c_{j R}-c_{j R}^{\dagger} c_{j L}\right), \\
S_{j}^{z}=\frac{1}{2}\left(c_{j L}^{\dagger} c_{j L}-c_{j R}^{\dagger} c_{j R}\right) .
\end{gathered}
$$

This shows that $S_{j}^{x}$ corresponds to tunneling transitions between the left and right locations, $S_{j}^{y}$ characterizes the Josephson current between these locations, and $S_{j}^{z}$ describes the population imbalance between the wells. Thus, the operator $S_{j}^{z}$ can be called the deformation operator, since, when atoms move to one of the wells, the spatial system symmetry is broken 
and the system becomes deformed. For a finite system, such a deformation leads to the change of its shape.

Substituting expansion (39) into Hamiltonian (36), we denote the matrix elements of the interaction potential as $A\left(r_{i j}\right), B\left(r_{i j}\right)$, and $C\left(r_{i j}\right)$, whose detailed definition can be found in Refs. [89,90,97] and where

$$
r_{i j} \equiv\left|\mathbf{r}_{i j}\right|, \quad \mathbf{r}_{i j} \equiv \mathbf{r}_{i}-\mathbf{r}_{j}
$$

Also, we introduce the following notation:

$$
\begin{gathered}
E_{0} \equiv \frac{1}{2 N} \sum_{j}\left(V_{j j}^{11}+V_{j j}^{22}\right), \quad p_{j}^{2} \equiv m\left(K_{j j}^{11}+K_{j j}^{22}\right), \\
\Omega_{j} \equiv E_{j j}^{22}-E_{j j}^{11}+\sum_{i} C\left(r_{i j}\right), \quad h_{j} \equiv E_{j j}^{12}+E_{j j}^{21}=U_{j j}^{12}+U_{j j}^{21} .
\end{gathered}
$$

In this way, Hamiltonian (36) transforms into

$$
\begin{gathered}
\hat{H}=N E_{0}+\sum_{j}\left(\frac{p_{j}^{2}}{2 m}-\Omega_{j} S_{j}^{x}+h_{j} S_{j}^{z}\right)+ \\
+\sum_{i \neq j}\left[\frac{1}{2} A\left(r_{i j}\right)+B\left(r_{i j}\right) S_{i}^{x} S_{j}^{x}-I\left(r_{i j}\right) S_{i}^{z} S_{j}^{z}\right] .
\end{gathered}
$$

Note that the expression $h_{j}$, playing the role of an external field, is nonzero only when the external potential $U_{e x t}(\mathbf{r}, t)$ is not uniform. As soon as $U_{e x t}$ is uniform in space, the effective field $h_{j}$ is zero.

\subsection{Operators of atomic deviations}

Taking into account vibrational degrees of freedom can be done by introducing the operators of atomic deviations $\mathbf{u}_{j}$ by the equation

$$
\mathbf{r}_{j}=\mathbf{a}_{j}+\mathbf{u}_{j}
$$

where $\mathbf{a}_{j}$ is a fixed vector related to a $j$-th lattice site. Since the interaction terms in Eq. (50) depend on the difference $\mathbf{r}_{i j}$, it is convenient to use the relative deviation

$$
\mathbf{u}_{i j} \equiv \mathbf{u}_{i}-\mathbf{u}_{j}
$$

Treating $\mathbf{u}_{j}$ as small deviations around $\mathbf{a}_{j}$, we expand the interaction terms in Hamiltonian (50) in powers of $\mathbf{u}_{i j}$. In this expansion, we use the notations

$$
A_{i j} \equiv A\left(a_{i j}\right), \quad A_{i j}^{\alpha} \equiv \frac{\partial A_{i j}}{\partial a_{i}^{\alpha}}, \quad A_{i j}^{\alpha \beta} \equiv \frac{\partial^{2} A_{i j}}{\partial a_{i}^{\alpha} \partial a_{j}^{\beta}},
$$

where

$$
a_{i j} \equiv\left|\mathbf{a}_{i j}\right|, \quad \mathbf{a}_{i j} \equiv \mathbf{a}_{i}-\mathbf{a}_{j} .
$$

Analogous notations are employed in the expansions of other interaction terms. 
Since the expression

$$
A \equiv \frac{1}{N} \sum_{i j} A_{i j}=\sum_{j} A_{i j}
$$

does not depend on the atomic indices, we have

$$
\begin{gathered}
\sum_{j} A_{i j}^{\alpha}=\frac{\partial A}{\partial a_{i}^{\alpha}}=0, \quad \sum_{j} A_{i j}^{\alpha \beta}=-\frac{\partial^{2} A}{\partial a_{i}^{\alpha} \partial a_{i}^{\beta}}=0, \\
\sum_{i j} A_{i j}^{\alpha} u_{i j}^{\alpha}=2 \sum_{i} u_{i}^{\alpha} \sum_{j} A_{i j}^{\alpha}=0 .
\end{gathered}
$$

In the above summations, we keep in mind that $i \neq j$, though this is not shown explicitly for the brevity of notation. Alternatively, we may set $A_{i i} \equiv 0, I_{i i} \equiv 0, B_{i i} \equiv 0$.

Following this way $[97,98]$, we come to the Hamiltonian

$$
\begin{gathered}
\hat{H}=\left(E_{0}+\frac{A}{2}\right) N+\sum_{j}\left(\frac{p_{j}^{2}}{2 m}-\Omega_{j} S_{j}^{x}+h_{j} S_{j}^{z}\right)+ \\
+\sum_{i j} \sum_{\alpha \beta}\left[-\frac{1}{4} A_{i j}^{\alpha \beta} u_{i j}^{\alpha} u_{i j}^{\beta}+\left(B_{i j}+\sum_{\alpha} B_{i j}^{\alpha} u_{i j}^{\alpha}-\frac{1}{2} \sum_{\alpha \beta} B_{i j}^{\alpha \beta} u_{i j}^{\alpha} u_{i j}^{\beta}\right) S_{i}^{x} S_{j}^{x}-\right. \\
\left.-\left(I_{i j}+\sum_{\alpha} I_{i j}^{\alpha} u_{i j}^{\alpha}-\frac{1}{2} \sum_{\alpha \beta} I_{i j}^{\alpha \beta} u_{i j}^{\alpha} u_{i j}^{\beta}\right) S_{i}^{z} S_{j}^{z}\right] .
\end{gathered}
$$

Vibrational and atomic degrees of freedom in the four-operator terms can be decoupled by the relation

$$
u_{i j}^{\alpha} u_{i j}^{\beta} S_{i}^{\gamma} S_{j}^{\gamma}=\left\langle u_{i j}^{\alpha} u_{i j}^{\beta}\right\rangle S_{i}^{\gamma} S_{j}^{\gamma}+u_{i j}^{\alpha} u_{i j}^{\beta}\left\langle S_{i}^{\gamma} S_{j}^{\gamma}\right\rangle-\left\langle u_{i j}^{\alpha} u_{i j}^{\beta}\right\rangle\left\langle S_{i}^{\gamma} S_{j}^{\gamma}\right\rangle,
$$

while keeping untouched the lower-order terms. We suppose that the pseudospin correlation functions $\left\langle S_{i}^{\alpha} S_{j}^{\alpha}\right\rangle$ weakly depend on the lattice indices, so that

$$
\begin{gathered}
\sum_{j(\neq i)} B_{i j}^{\alpha \beta}\left\langle S_{i}^{x} S_{j}^{x}\right\rangle \cong-\frac{\partial^{2}}{\partial a_{i}^{\alpha} \partial a_{i}^{\beta}} \sum_{j(\neq i)} B_{i j}\left\langle S_{i}^{x} S_{j}^{x}\right\rangle \cong 0, \\
\sum_{j(\neq i)} I_{i j}^{\alpha \beta}\left\langle S_{i}^{z} S_{j}^{z}\right\rangle \cong-\frac{\partial^{2}}{\partial a_{i}^{\alpha} \partial a_{i}^{\beta}} \sum_{j(\neq i)} I_{i j}\left\langle S_{i}^{z} S_{j}^{z}\right\rangle \cong 0 .
\end{gathered}
$$

Thus we come to the Hamiltonian

$$
\hat{H}=\widetilde{E}_{0} N+H_{p h}+H_{a t}+H_{\text {int }} .
$$

Here, in the first term

$$
\widetilde{E}_{0} \equiv E_{0}+\frac{A}{2}+\frac{1}{N} \sum_{i j} \sum_{\alpha \beta}\left(I_{i j}^{\alpha \beta}\left\langle S_{i}^{z} S_{j}^{z}\right\rangle-B_{i j}^{\alpha \beta}\left\langle S_{i}^{x} S_{j}^{x}\right\rangle\right)\left\langle u_{i}^{\alpha} u_{j}^{\beta}-u_{j}^{\alpha} u_{j}^{\beta}\right\rangle .
$$


The second term is the effective Hamiltonian of dressed phonons

$$
H_{p h}=\sum_{j} \frac{p_{j}^{2}}{2 m}+\frac{1}{2} \sum_{i j} \sum_{\alpha \beta} \Phi_{i j}^{\alpha \beta} u_{i}^{\alpha} u_{j}^{\beta}
$$

with the renormalized dynamic matrix

$$
\Phi_{i j}^{\alpha \beta} \equiv A_{i j}^{\alpha \beta}-2 I_{i j}^{\alpha \beta}\left\langle S_{i}^{z} S_{j}^{z}\right\rangle+2 B_{i j}^{\alpha \beta}\left\langle S_{i}^{x} S_{j}^{x}\right\rangle
$$

The third term is the Hamiltonian of dressed atoms

$$
H_{a t}=\sum_{j}\left(h_{j} S_{j}^{z}-\Omega_{j} S_{j}^{x}\right)+\sum_{i j}\left(\widetilde{B}_{i j} S_{i}^{x} S_{j}^{x}-\widetilde{I}_{i j} S_{i}^{z} S_{j}^{z}\right)
$$

with the renormalized atomic interactions

$$
\widetilde{B}_{i j} \equiv B_{i j}+\sum_{\alpha \beta} B_{i j}^{\alpha \beta}\left\langle u_{i}^{\alpha} u_{j}^{\beta}-u_{j}^{\alpha} u_{j}^{\beta}\right\rangle, \quad \widetilde{I}_{i j} \equiv I_{i j}+\sum_{\alpha \beta} I_{i j}^{\alpha \beta}\left\langle u_{i}^{\alpha} u_{j}^{\beta}-u_{j}^{\alpha} u_{j}^{\beta}\right\rangle .
$$

And the fourth term describes atom-phonon interactions,

$$
H_{\text {int }}=-2 \sum_{i} \sum_{\alpha} F_{i}^{\alpha} u_{i}^{\alpha}
$$

in which the effective force acting on an atom is

$$
F_{i}^{\alpha} \equiv \sum_{j} F_{i j}^{\alpha}
$$

where

$$
F_{i j}^{\alpha} \equiv I_{i j}^{\alpha} S_{i}^{z} S_{j}^{z}-B_{i j}^{\alpha} S_{i}^{x} S_{j}^{x}
$$

\subsection{Pseudophonon and phonon operators}

In order to introduce phonon operators, let us define in the standard way the phonon frequency $\omega_{k s}$ by the eigenproblem

$$
\frac{1}{m} \sum_{j} \sum_{\beta} \Phi_{i j}^{\alpha \beta} e^{i \mathbf{k} \cdot \mathbf{a}_{i j}} e_{k s}^{\beta}=\omega_{k s}^{2} e_{k s}^{\alpha}
$$

where $\mathbf{k}$ is momentum, $s=1,2,3$ is polarization index, and $\mathbf{e}_{k s}$ is a polarization vector.

If one defines the phonon operators $d_{k s}$ by the usual relations

$$
\begin{gathered}
\mathbf{p}_{j}=-\frac{i}{\sqrt{2 N}} \sum_{k s} \sqrt{m \omega_{k s}} \mathbf{e}_{k s}\left(d_{k s}-d_{-k s}^{\dagger}\right) e^{i \mathbf{k} \cdot \mathbf{a}_{j}} \\
\mathbf{u}_{j}=\frac{1}{\sqrt{2 N}} \sum_{k s} \frac{\mathbf{e}_{k s}}{\sqrt{m \omega_{k s}}}\left(d_{k s}+d_{-k s}^{\dagger}\right) e^{i \mathbf{k} \cdot \mathbf{a}_{j}}
\end{gathered}
$$


the total Hamiltonian (55) does not become diagonal with respect to these operators $d_{k s}$. That is, such operators are not true phonon operators, but rather some pseudophonon operators. To diagonalize the Hamiltonian, we need to accomplish an additional canonical transformation

$$
d_{k s}=b_{k s}+\frac{2}{\sqrt{2 N} \omega_{k s} \sqrt{m \omega_{k s}}} \sum_{i} \sum_{\alpha} F_{i}^{\alpha} e_{k s}^{\alpha} e^{-i \mathbf{k} \cdot \mathbf{a}_{i}} .
$$

This is equivalent [97] to introducing the phonon operators $b_{k s}$ by the nonuniform relations

$$
\begin{aligned}
& \mathbf{p}_{j}=-\frac{i}{\sqrt{2 N}} \sum_{k s} \sqrt{m \omega_{k s}} \mathbf{e}_{k s}\left(b_{k s}-b_{-k s}^{\dagger}\right) e^{i \mathbf{k} \cdot \mathbf{a}_{j}}, \\
& \mathbf{u}_{j}=\boldsymbol{\Delta}_{j}+\frac{1}{\sqrt{2 N}} \sum_{k s} \frac{\mathbf{e}_{k s}}{\sqrt{m \omega_{k s}}}\left(b_{k s}+b_{-k s}^{\dagger}\right) e^{i \mathbf{k} \cdot \mathbf{a}_{j}},
\end{aligned}
$$

where the additional vector term has the components

$$
\Delta_{i}^{\alpha}=2 \sum_{j} \sum_{\beta} \gamma_{i j}^{\alpha \beta} F_{j}^{\beta}
$$

with the matrix

$$
\gamma_{i j}^{\alpha \beta} \equiv \frac{1}{N} \sum_{k s} \frac{e_{k s}^{\alpha} e_{k s}^{\beta}}{m \omega_{k s}^{2}} e^{i \mathbf{k} \cdot \mathbf{a}_{i j}} .
$$

As a result of this nonuniform transformation (67), Hamiltonian (55) takes the form

$$
\hat{H}=N \widetilde{E}_{0}+\widetilde{H}_{p h}+H_{a t}+H_{4}
$$

with the diagonal phonon part

$$
\widetilde{H}_{p h}=\sum_{k s} \omega_{k s}\left(b_{k s}^{\dagger} b_{k s}+\frac{1}{2}\right)
$$

and with an additional four-atom interaction term

$$
H_{4}=-\frac{4}{N} \sum_{i j} \sum_{f g} \sum_{\alpha \beta} F_{i j}^{\alpha} \gamma_{j f}^{\alpha \beta} F_{f g}^{\beta} .
$$

Since the Hamiltonian is diagonal in operators $b_{k s}$, it is these operators that should be accepted as real phonon operators.

\subsection{Shape distortion operator}

Operator (68), entering transformation (67), characterizes the shift of the deviation operator $\mathbf{u}_{j}$ caused by the existence of atomic degrees of freedom described by the pseudospin operators $S_{j}^{\alpha}$. Such a shift of atoms from their initial positions $\mathbf{a}_{j}$ would lead to the distortion of the considered sample, because of which the operator $\boldsymbol{\Delta}_{j}$ can be called the distortion operator. In the case of a finite system, this deformation results in the change of the sample shape. To illustrate more explicitly the form of the distortion operator, one needs to specify the phonon frequency. 
If we take the phonon frequency in the Einstein approximation

$$
\omega_{k s}=\omega_{0},
$$

then matrix (69) is

$$
\gamma_{i j}^{\alpha \beta}=\frac{\delta_{\alpha \beta} \delta_{i j}}{m \omega_{0}^{2}} .
$$

This gives the distortion operator

$$
\Delta_{i}^{\alpha}=\frac{2}{m \omega_{0}^{2}} F_{i}^{\alpha} .
$$

Another common case is the Debye approximation for the frequency

$$
\omega_{k s}=\Theta\left(k_{D}-k\right) c k,
$$

in which $\Theta(k)$ is a unit step function and

$$
k_{D} \equiv\left(6 \pi^{2} \rho\right)^{1 / 3}
$$

is the Debye wave vector modulus. Then for matrix (69), we get

$$
\gamma_{i j}^{\alpha \beta}=\frac{\delta_{\alpha \beta}}{2 \pi^{2} \rho m c^{2}} \frac{\operatorname{Si}\left(k_{D} a_{i j}\right)}{a_{i j}},
$$

where $\operatorname{Si}(x)$ is the sine integral

$$
\operatorname{Si}(x) \equiv \int_{0}^{x} \frac{\sin t}{t} d t .
$$

The latter possesses the asymptotic properties

$$
\begin{gathered}
\operatorname{Si}(x) \simeq x-\frac{x^{3}}{18} \quad(x \rightarrow 0), \\
\operatorname{Si}(x) \simeq \frac{\pi}{2}-\frac{\cos x}{x} \quad(x \rightarrow \infty) .
\end{gathered}
$$

The distortion operator becomes

$$
\Delta_{i}^{\alpha}=\frac{1}{\pi^{2} \rho m c^{2}} \sum_{j} F_{j}^{\alpha} \frac{\operatorname{Si}\left(k_{D} a_{i j}\right)}{a_{i j}} .
$$

\subsection{No equilibrium phonon condensation}

Real phonons are not conserved quasiparticles and, as is seen from the phonon Hamiltonian (71),

$$
\left\langle b_{k s}\right\rangle=0,
$$

that is, equilibrium phonons cannot condense. At the same time, as follows from Eq. (67), the average deviation can be nonzero, since

$$
\left\langle\mathbf{u}_{j}\right\rangle=\left\langle\boldsymbol{\Delta}_{j}\right\rangle .
$$


To explicitly show how the deviation can arise, let us resort to the mean-field approximation for the correlation function

$$
\left\langle S_{i}^{\alpha} S_{j}^{\alpha}\right\rangle=\left\langle S_{i}^{\alpha}\right\rangle\left\langle S_{j}^{\alpha}\right\rangle \quad(i \neq j)
$$

Denoting the mean pseudospin

$$
C_{J}^{\alpha} \equiv\left\langle S_{j}^{\alpha}\right\rangle
$$

we have for the average force (62)

$$
\left\langle F_{i}^{\alpha}\right\rangle=\sum_{j}\left(I_{i j}^{\alpha} C_{i}^{z} C_{j}^{z}-B_{i j}^{\alpha} C_{i}^{x} C_{j}^{x}\right)
$$

Therefore, the observed distortion (68) is, generally, nonzero. For instance, taking the phonon frequency in the Einstein approximation yields

$$
\left\langle\Delta_{i}^{\alpha}\right\rangle=\frac{2}{m \omega_{0}^{2}} \sum_{j}\left(I_{i j}^{\alpha} C_{i}^{z} C_{j}^{z}-B_{i j}^{\alpha} C_{i}^{x} C_{j}^{x}\right) .
$$

When $C_{j}^{\alpha}$ is zero itself or does not depend on the index $j$, then distortion (82) is zero. But for $C_{j}^{\alpha} \neq 0$ and depending on $j$, there appears a nonzero distortion. For double wells, the average $C_{j}^{z}$ plays the role of an order parameter distinguishing a disordered phase with $C_{j}^{z}=0$ from an ordered phase where $C_{j}^{z} \neq 0$. The ordered phase exists at low temperatures below a critical temperature $[89,90]$. The ordering means the appearance of atomic imbalance between the wells of a double well. The order-disorder phase transition occurs if there is no external force $h_{j}$ defined in Eq. (49). When the latter is absent, the system Hamiltonian (55) is invariant under the inversion $S_{j}^{z} \rightarrow-S_{j}^{z}$, which implies the existence of a degenerate ground state. This situation is analogous to the Jahn-Teller effect $[99,100]$, where the existence of a degenerate ground state of a molecule leads to the distortion of the latter.

The appearance of a nonzero distortion (82) could be associated with the condensation of pseudophonons described by the operators $d_{k s}$. However, it is evident that this condensation simply means the shift of the average location of each atom occurring at the point of the order-disorder phase transition. At this point the system is unstable. After the phase transition has happened, one has to change the definition of atomic locations and, respectively, the definition of the deviation from this location.

Alternatively, it is possible to define the atomic locations as the averages

$$
\mathbf{a}_{j} \equiv\left\langle\mathbf{r}_{j}\right\rangle
$$

Then, in view of relation (51), one always has zero deviation

$$
\left\langle\mathbf{u}_{j}\right\rangle=0
$$

and, because of equality (78), zero distortion

$$
\left\langle\boldsymbol{\Delta}_{j}\right\rangle=0
$$

In this case, the occurrence of the order-disorder phase transition is exhibited in the sharp variation, e.g., with temperature, of the average atomic location (83). But there is neither phonon nor pseudophonon condensation. 
Condition (84) can be preserved in any given approximation by defining the grand Hamiltonian

$$
H=\hat{H}-\sum_{j} \vec{\lambda}_{j} \cdot \mathbf{u}_{j}
$$

in which the Lagrange multipliers $\lambda_{j}$ are chosen so that to cancel the linear in the deviations $\mathbf{u}_{j}$ terms, thus, guaranteeing the statistical condition (84).

Even more, definition (83) can be extended to nonequilibrium systems by introducing atomic deviations through the equation

$$
\mathbf{r}_{j}(t)=\mathbf{a}_{j}(t)+\mathbf{u}_{j}(t)
$$

in which the first term is the average

$$
\mathbf{a}_{j}(t) \equiv\left\langle\mathbf{r}_{j}(t)\right\rangle
$$

I that case, by definition,

$$
\left\langle\mathbf{u}_{j}(t)\right\rangle=0
$$

hence there is no symmetry breaking:

$$
\left\langle b_{k s}(t)\right\rangle=0 \text {. }
$$

Defining in such a self-consistent way atomic deviations makes phonon condensation absent in any nonequilibrium system.

\section{No equilibrium magnon condensation}

Collective excitations, characterizing spin oscillations, or spin waves, are magnons. Their definition can be done by the Holstein-Primakoff transformation [101,102] for the ladder spin operators

$$
S_{j}^{ \pm} \equiv S_{j}^{x} \pm i S_{j}^{y} .
$$

This transformation for the spin operator $\mathbf{S}_{j}$, located at $\mathbf{r}_{j}$, reads as

$$
\begin{gathered}
S_{j}^{+}=\sqrt{2 S-b_{j}^{\dagger} b_{j}} b_{j}, \quad S_{j}^{-}=b_{j}^{\dagger} \sqrt{2 S-b_{j}^{\dagger} b_{j}}, \\
S_{j}^{z}=S-b_{j}^{\dagger} b_{j},
\end{gathered}
$$

where $S$ is the spin value and $b_{j}$ are Bose operators representing magnons. The square root of operators is to be understood as Taylor series in powers of the magnon density operator

$$
\hat{n}_{j} \equiv b_{j}^{\dagger} b_{j}
$$

which implies the assumption on the small deviation $S-S_{j}^{z}$. The eigenvalues of the magnondensity operator $\hat{n}_{j}$ are $0,1,2, \ldots, 2 S$. In the lowest approximation,

$$
S_{j}^{+} \simeq \sqrt{2 S} b_{j}, \quad S_{j}^{-} \simeq \sqrt{2 S} b_{j}^{+} .
$$


The Holstein-Primakoff magnons, by definition, characterize small spin fluctuations around the ground state, for which the average spin is directed along the $z$-axis,

$$
\left\langle\mathbf{S}_{j}\right\rangle=S \mathbf{e}_{z}
$$

so that there are no transverse components:

$$
\left\langle S_{j}^{x}\right\rangle=\left\langle S_{j}^{y}\right\rangle=0
$$

The latter means that

$$
\left\langle b_{j}\right\rangle=0 \text {. }
$$

By construction, the Holstein-Primakoff representation (86) presupposes that magnons describe small fluctuations around the saturated magnetization directed along the $z$-axis [101]. The appearance of magnon condensation would mean the rotation of the magnetization because of the arising nonzero transverse magnetization. But then the total magnetization is not directed anymore along the $z$-axis, as is assumed in the Holstein-Primakoff representation (86). That is, the latter is not applicable, so that magnons are not well defined.

If in calculations one formally meets magnon condensation in an equilibrium state, when $\left\langle b_{j}\right\rangle$ becomes nonzero, this implies that at that point a phase transition occurs, with the rotation of the average magnetization. Then one has to redefine the system ground state, for which the Holstein-Primakoff transformation (86) would be valid, since representation (86) is not applicable if magnons would be condensed. The rotation of system magnetization does not mean magnon condensation, but means the change of the system ground state.

Magnons are not conserved and in equilibrium they cannot condense, $\left\langle b_{j}\right\rangle=0$, similarly to the impossibility of equilibrium phonon condensation or to the equilibrium condensation of other unconserved quasiparticles. Impossibility of the equilibrium magnon condensation has been stressed by Mills [36].

There are interpretations of some magnetic effects in nonequilibrium systems as being due to magnon condensation. These can be the systems that are subject to a sufficiently strong external alternating field, whose frequency plays the role of a magnon chemical potential determining the nonequilibrium density of magnons [103-108]. Or these can be the spin systems prepared in a strongly nonequilibrium initial state and relaxing to equilibrium by essentially nonlinear dynamics [109-120]. The magnon condensation is manifested as the emerging state of precessing spins, where all spins precess with the same frequency and phase. This nonequilibrium magnon condensation is accompanied by the arising off-diagonal order, when the averages

$$
\left\langle S_{j}^{ \pm}\right\rangle \propto e^{i \omega_{0} t}
$$

are nonzero, where $\omega_{0}$ is the Zeeman frequency. The nonequilibrium magnon condensation is an example of dynamic coherence, contrary to static coherence related to the Bose-Einstein condensation of conserved atoms.

As is mentioned in the Introduction, there exists a number of articles claiming the occurrence of magnon condensation in equilibrium stable magnets. Such claims are based on confusion, when either the Holstein-Primakoff representation is used outside of its region of validity or transformations are employed to some auxiliary quasiparticles that have nothing to do with magnons. One could talk on magnon condensation at magnetic phase transitions [121-123], which does not contradict the above conclusion on the impossibility of equilibrium 
magnon condensation, since the point of a phase transition is that where the system loses its stability, hence, an equilibrium system does not exist. But the occurrence of magnon condensation in the whole range of thermodynamic parameters, in an equilibrium system, is principally impossible.

\section{Operators of auxiliary quasiparticles}

I many cases, one accomplishes operator canonical transformations aiming at simplifying the solution of a problem. In such transformations, the newly introduced operators correspond to auxiliary quiasiparticles that do not necessarily represent physical particles, but can be just mathematical tools, not related to any physical objects. In many cases the statistics of such auxiliary quasiparticles are even not uniquely defined, and the latter can be equally treated as bosons or as fermions. Depending on whether the auxiliary quasiparticles are conserved and prescribed to be bosons, they can formally exhibit condensation in equilibium systems. In this and in the following section, some examples of auxiliary quasiparticles are given.

\subsection{Jordan-Wigner transformation}

As an example of a trasformation, introducing auxiliary quasiparticle, let us mention the mapping of spin operators of spin $1 / 2$, for a one-dimensional chain, onto the fermionic operators. After this mapping, one expresses the fermionic operators in terms of bosonic ones using Abelian bosonization [124,125].

One considers the spin operators

$$
\mathbf{S}_{j}=\frac{1}{2} \vec{\sigma}_{j}
$$

where $\vec{\sigma}_{j}$ is the vector Pauli matrix. The Jordan-Wigner [126] transformation

$$
S_{j}^{+}=\exp \left(i \pi \sum_{n=1}^{j-1} a_{n}^{\dagger} a_{n}\right) a_{j}^{\dagger}, \quad S_{j}^{-}=\exp \left(-i \pi \sum_{n=1}^{j-1} a_{n}^{\dagger} a_{n}\right) a_{j}, \quad S_{j}^{z}=a_{j}^{\dagger} a_{j}-\frac{1}{2},
$$

maps the ladder spin operators onto fermionic operators $a_{j}$. The latter do not correspond to some really existing particles or quasiparticles, but they just allow one to solve the studied one-dimensional problem.

One may notice that

$$
S_{j}^{+} S_{j}^{-}=a_{j}^{\dagger} a_{j}
$$

Thence, the last of transformations (92) can be written in the form

$$
S_{j}^{z}=S_{j}^{+} S_{j}^{-}-\frac{1}{2} .
$$

This is in agreement with the general properties of $S$-spin operators

$$
\mathbf{S}_{i} \cdot \mathbf{S}_{j}=\frac{1}{2}\left(S_{i}^{+} S_{j}^{-}+S_{j}^{+} S_{i}^{-}\right)+S_{i}^{z} S_{j}^{z}-\delta_{i j} S_{j}^{z},
$$




$$
\mathbf{S}_{j}^{2}=S_{j}^{+} S_{j}^{-}+\left(S_{j}^{z}\right)^{2}-S_{j}^{z}=S(S+1) .
$$

The inverse transformation reads as

$$
a_{j}^{+}=\exp \left(-i \pi \sum_{n=1}^{j-1} S_{n}^{+} S_{n}^{-}\right) S_{j}^{+}, \quad a_{j}^{-}=\exp \left(i \pi \sum_{n=1}^{j-1} S_{n}^{+} S_{n}^{-}\right) S_{j}^{-} .
$$

The total number of the auxiliary quasiparticles is not conserved, since

$$
\sum_{j}\left\langle a_{j}^{\dagger} a_{j}\right\rangle=\frac{N}{2}+\sum_{j}\left\langle S_{j}^{z}\right\rangle
$$

In this case, the auxiliary fermionic operators do not represent physical particles. If in the process of some canonical transformation one would employ a mapping to auxiliary bosonic operators, one could talk on the possible condensation of the related auxiliary quasiparticles. Recall, however, that equilibrium condensation can happen only for conserved quasiparticles.

\subsection{Condensation of Schwinger bosons}

Auxiliary quasiparticles can be introduced, for example, by means of the transformation

$$
\mathbf{S}_{j}=\frac{1}{2} \sum_{\alpha \beta} a_{j \alpha}^{\dagger} \vec{\sigma}_{\alpha \beta} a_{j \beta}
$$

mapping the spin operator $\mathbf{S}_{j}$ at the spatial location $\mathbf{r}_{j}$ to quasiparticle operators $a_{j \alpha}$, in which $\alpha=1,2$ enumerates two admissible states, for instance, spin projections. The operator

$$
\vec{\sigma}_{\alpha \beta}=\sum_{\gamma} \sigma_{\alpha \beta}^{\gamma} \mathbf{e}_{\gamma} \quad(\gamma=x, y, z)
$$

is a linear combination of the elements $\sigma_{\alpha \beta}^{\gamma}$ of the Pauli matrices, where $\mathbf{e}_{\gamma}$ is a unit vector in the direction labelled by $\gamma=x, y, z$. Thus, the components of vector (96) are

$$
S_{j}^{\gamma}=\frac{1}{2} \sum_{\alpha \beta} a_{j \alpha}^{\dagger} \sigma_{\alpha \beta}^{\gamma} a_{j \beta}
$$

Explicitly, this transformation takes the form

$$
S_{j}^{+}=a_{j 1}^{\dagger} a_{j 2}, \quad S_{j}^{-}=a_{j 2}^{\dagger} a_{j 1}, \quad S_{j}^{z}=\frac{1}{2}\left(a_{j 1}^{\dagger} a_{j 1}-a_{j 2}^{\dagger} a_{j 2}\right) .
$$

The quasiparticle operators satisfy the constraint

$$
\sum_{\alpha} a_{j \alpha}^{\dagger} a_{j \alpha}=2 S
$$

It is important to emphasize that $a_{j \alpha}$ can be either bosonic or fermionic operators, thus, representing any type of quasiparticles, whether bosons or fermions. Transformation (98) was, first, introduced by Bogolubov $[62,127]$, with the operators $a_{j \alpha}$ representing electrons, that is, fermions. Schwinger [128] treated $a_{j \alpha}$ as representing bosons, because of which the 
latter are called the Schwinger bosons [129]. If the index $\alpha$ is interpreted as spin projection, then the Schwinger bosons are fictitious quasiparticles that cannot exist in reality, which follows from their unphysical nature, as far as they are bosons possessing spin one-half. But they can be real particles when the index $\alpha$ corresponds to the enumeration of some other components. Also, pairons [130], that are tightly bound pairs of particles, could be connected with Schwinger bosons [131].

In view of constraint (99), the total number of quasiparticles in conserved:

$$
\sum_{j} \sum_{\alpha}\left\langle a_{j \alpha}^{\dagger} a_{j \alpha}\right\rangle=2 S N
$$

Being treated as bosons, conserved quasiparticles can condense, which has been used in the interpretation of magnetic transitions [132,133], though interpreting the magnetic orderdisorder phase transition as the Bose-Einstein condensation of Schwinger bosons can lead to incorrect first-order transition, instead of the correct second-order one [134].

Schwinger bosons are conserved quasiparticles and are principally different from the unconserved Holstein-Primakoff magnons [134]. This is why the former can condense in equilibrium systems, while the latter cannot.

\subsection{Auxiliary slave bosons}

In the theory of strongly correlated Fermi systems, one uses the slave-particle approach based on the factorization of the electron field operator into two operators, one of which is bosonic and another, fermionic. There are two alternatives that are termed slave boson and slave fermion representations.

In the slave boson representation [135-137], the electron operator $c_{i \sigma}$, describing the annihilation of an electron at the point $\mathbf{r}_{i}$ with spin $\sigma$, is formally split into the product

$$
c_{i \sigma}=b_{i}^{\dagger} f_{i \sigma}
$$

where $b_{i}$ is a spinless bosonic operator and $f_{i \sigma}$ is a fermionic operator with spin $\sigma$. The operators satisfy the no-double-occupancy constraint

$$
b_{i}^{\dagger} b_{i}+\sum_{\sigma} f_{i \sigma}^{\dagger} f_{i \sigma}=1
$$

The bosonic operator $b_{i}$ is said to represent an auxiliary charged spinless quasiparticle, named holon, while the fermionic operator $f_{i \sigma}$, an auxiliary neutral spin one-half quasiparticle spinon.

By this definition, it is clear that the holons and spinons are fictitious quasiparticles. According to constraint (102), the total number of these auxiliary quasiparticles is conserved:

$$
\sum_{i}\left(\left\langle b_{i}^{\dagger} b_{i}\right\rangle+\sum_{\sigma}\left\langle f_{i \sigma}^{\dagger} f_{i \sigma}\right\rangle\right)=N,
$$

where $N$ is the total number of electrons. The number of holons separately, strictly speaking, is not conserved. But it can be treated as quasiconserved, when the number of spinons is limited by a value smaller than $N$. If the number of spinons is fixed by the doping 
concentration, as is usually done, then holons become conserved exactly. Therefore, it is admissible to talk about holon condensation [138] or holon pair condensation [139].

In the slave fermion representation [140-142], one formally splits the electron operator into the product

$$
c_{i \sigma}=f_{i}^{+} b_{i \sigma}
$$

with a fermionic operator $f_{i}$ corresponding to spinless charged fermionic holons and a bosonic operator $b_{i \sigma}$ describing neutral spin one-half bosonic spinons. Again, the no-doubleoccupancy constraint is imposed:

$$
\sum_{\sigma} b_{i \sigma}^{\dagger} b_{i \sigma}+f_{i}^{+} f_{i}=1
$$

so that the total number of holons and spinons is conserved,

$$
\sum_{i}\left(\sum_{\sigma}\left\langle b_{i \sigma}^{\dagger} b_{i \sigma}\right\rangle+\left\langle f_{i}^{+} f_{i}\right\rangle\right)=N,
$$

being normalized to the total number of electrons. Here, the spinons are rather unphysical quasiparticles, being bosons with spin one-half.

Fictitious quasiparticles, introduced by the slave particle representations, are not real particles, being just auxiliary tools for calculations. In these calculations, one has to preserve the imposed constraints that limit the efficiency of the representations [143]. Under conditions, when the bosonic quasiparticles can be made conserved, they are allowed for formally experiencing condensation.

\section{Condensation of singletons and triplons}

Auxiliary quasiparticles are also introduced in the theory of quantum antiferromagnets, as suggested by Sachdev and Bhatt [144]. Generally, these quasiparticles can be defined either as bosons or as fermions. Treating them as bosons makes their condensation possible, provided they are conserved.

\subsection{Bond-operator representation}

Suppose there are two spins one-half, with the related operators $\mathbf{S}_{1}$ and $\mathbf{S}_{2}$. This pair of spins can be treated as a dimer connected by bonds. Sachdev and Bhatt [144] suggested for the pair of the spins the bond-operator representation

$$
\begin{gathered}
S_{1}^{\alpha}=\frac{1}{2}\left(s^{\dagger} t_{\alpha}+t_{\alpha}^{\dagger} s-i \sum_{\beta \gamma} \varepsilon_{\alpha \beta \gamma} t_{\beta}^{\dagger} t_{\gamma}\right), \\
S_{2}^{\alpha}=-\frac{1}{2}\left(s^{\dagger} t_{\alpha}+t_{\alpha}^{\dagger} s+i \sum_{\beta \gamma} \varepsilon_{\alpha \beta \gamma} t_{\beta}^{\dagger} t_{\gamma}\right),
\end{gathered}
$$

expressing the spins through the quasiparticle operator $s$, characterizing the singlet state, and the operators $t_{\alpha}$, with $\alpha=x, y, z$, describing the triplet state. Here, $\varepsilon_{\alpha \beta \gamma}$ is the totally 
antisymmetric unit tensor. The corresponding auxiliary quasiparticles can, therefore, be called singletons, and triplons, respectively. Explicitly, Eqs. (106) read as

$$
\begin{gathered}
S_{1}^{x}=\frac{1}{2}\left[s^{\dagger} t_{x}+t_{x}^{\dagger} s-i\left(t_{y}^{\dagger} t_{z}-t_{z}^{\dagger} t_{y}\right)\right], \quad S_{1}^{y}=\frac{1}{2}\left[s^{\dagger} t_{y}+t_{y}^{\dagger} s-i\left(t_{z}^{\dagger} t_{x}-t_{x}^{\dagger} t_{z}\right)\right], \\
S_{1}^{z}=\frac{1}{2}\left[s^{\dagger} t_{z}+t_{z}^{\dagger} s-i\left(t_{x}^{\dagger} t_{y}-t_{y}^{\dagger} t_{x}\right)\right],
\end{gathered}
$$

for the first spin, and as

$$
\begin{gathered}
S_{2}^{x}=-\frac{1}{2}\left[s^{\dagger} t_{x}+t_{x}^{\dagger} s+i\left(t_{y}^{\dagger} t_{z}-t_{z}^{\dagger} t_{y}\right)\right], \quad S_{2}^{y}=-\frac{1}{2}\left[s^{\dagger} t_{y}+t_{y}^{\dagger} s+i\left(t_{z}^{\dagger} t_{x}-t_{x}^{\dagger} t_{z}\right)\right], \\
S_{2}^{z}=-\frac{1}{2}\left[s^{\dagger} t_{z}+t_{z}^{\dagger} s+i\left(t_{x}^{\dagger} t_{y}-t_{y}^{\dagger} t_{x}\right)\right],
\end{gathered}
$$

for the second spin. This representation could be considered as a generalized Schwinger representation (96) extended to the pair of spins.

The restriction on the physical states to be either singlets or triplets leads to the constraint

$$
s^{\dagger} s+\sum_{\alpha} t_{\alpha}^{\dagger} t_{\alpha}=1
$$

with $\alpha=x, y, z$.

The spin operators satisfy the standard commutation relations

$$
\left[S_{1}^{\alpha}, S_{1}^{\beta}\right]=i \sum_{\gamma} \varepsilon_{\alpha \beta \gamma} S_{1}^{\gamma}, \quad\left[S_{2}^{\alpha}, S_{2}^{\beta}\right]=i \sum_{\gamma} \varepsilon_{\alpha \beta \gamma} S_{2}^{\gamma}, \quad\left[S_{1}^{\alpha}, S_{2}^{\beta}\right]=0
$$

and are normalized as

$$
\mathbf{S}_{1}^{2}=\mathbf{S}_{2}^{2}=S(S+1)=\frac{3}{4} .
$$

Under these conditions, the correct algebra of spin operators can be reproduced by either bosonic or fermionic commutation relations for the operators of the auxiliary quasiparticles. The statistics of these quasiparticles is not uniquely defined; they can be either bosons or fermions. If one interprets them as bosons, then the bosonic commutation relations are assumed:

$$
\left[s, s^{\dagger}\right]=1, \quad\left[t_{\alpha}, t_{\beta}^{\dagger}\right]=\delta_{\alpha \beta}, \quad\left[s, t_{\alpha}\right]=\left[s, t_{\alpha}^{\dagger}\right]=0 .
$$

The scalar product of two different spin operators is

$$
\mathbf{S}_{1} \cdot \mathbf{S}_{2}=\frac{1}{4}\left(\sum_{\alpha} t_{\alpha}^{\dagger} t_{\alpha}-s^{\dagger} s\right)
$$

and the summary $z$-component is

$$
S_{1}^{z}+S_{2}^{z}=-i\left(t_{x}^{\dagger} t_{y}-t_{y}^{\dagger} t_{x}\right)
$$

One introduces the following canonical transformation

$$
t_{x}=\frac{1}{\sqrt{2}}\left(t_{+}+t_{-}\right), \quad t_{y}=-\frac{i}{\sqrt{2}}\left(t_{+}-t_{-}\right), \quad t_{z}=t_{0} .
$$


The new operators satisfy the bosonic commutation relations

$$
\left[t_{\mu}, t_{\nu}^{\dagger}\right]=\delta_{\mu \nu}, \quad\left[t_{\mu}, t_{\nu}\right]=0
$$

where $\mu, \nu=+,-, 0$

The constraint (107) takes the form

$$
s^{\dagger} s+\sum_{\nu} t_{\nu}^{\dagger} t_{\nu}=1
$$

with $\nu=+,-, 0$. The $z$-component sum (112) becomes

$$
S_{1}^{z}+S_{2}^{z}=t_{-}^{\dagger} t_{-}-t_{+}^{\dagger} t_{+} .
$$

\subsection{Lattice of dimers}

A lattice, consisting of two spin sublattices corresponding to two components, can be treated as a lattice of dimers. Each pair of spins in a dimer can be described as is explained in the previous subsection. Constraint (115) takes place at each lattice site enumerated by the index $j=1,2, \ldots, N$,

$$
s_{j}^{\dagger} s_{j}+\sum_{\nu} t_{j \nu}^{\dagger} t_{j \nu}=1
$$

where $\nu=+,-, 0$. This implies that the total number of quasiparticles is conserved:

$$
\sum_{j}\left(\left\langle s_{j}^{\dagger} s_{j}\right\rangle+\sum_{\nu}\left\langle t_{j \nu}^{\dagger} t_{j \nu}\right\rangle\right)=N .
$$

One usually assumes [144] that the singletons are completely condensed, so that $s_{j}$ can be replaced by the nonoperator real quantity $s$ equal to the average

$$
s=\left\langle s_{j}\right\rangle,
$$

which does not depend on the site index because of the ideality of the lattice.

Another standard assumption is that the main contribution among the triplons is due to only one type of triplons denoted by

$$
t_{j} \equiv t_{j+}
$$

Under these assumptions, the bond-operator representation reduces to

$$
\begin{gathered}
S_{1 j}^{x}=\frac{s}{2 \sqrt{2}}\left(t_{j}^{\dagger}+t_{j}\right)=-2 S_{2 j}^{x}, \quad S_{1 j}^{y}=\frac{i s}{2 \sqrt{2}}\left(t_{j}^{\dagger}-t_{j}\right)=-2 S_{2 j}^{y}, \\
S_{1 j}^{z}=-\frac{1}{2} t_{j}^{\dagger} t_{j}=S_{2 j}^{z} .
\end{gathered}
$$

And the normalization condition (118) becomes

$$
N s^{2}+\sum_{j}\left\langle t_{j}^{\dagger} t_{j}\right\rangle=N .
$$

Taking account of the lattice periodicity simplifies normalization (122) to

$$
s^{2}+\left\langle t_{j}^{\dagger} t_{j}\right\rangle=1 \text {. }
$$

The number of singletons is defined self-consistently by requiring the system stability. Then triplons are conserved quasiparticles that can experience Bose-Einstein condensation. 


\subsection{Order parameters}

There can exist two phases in the system, the normal phase of uncondensed triplons and that of condensed triplons [144]. The order parameter distinguishing these phases is the average $\left\langle t_{j}\right\rangle$. The normal phase, where triplons are not condensed, corresponds to magnetically disordered phase, while the condensed triplon phase is magnetically ordered [144]. The second order parameter is the absolute value of magnetization

$$
M \equiv \frac{1}{N}\left|\sum_{j}\left\langle S_{1 j}^{z}+S_{2 j}^{z}\right\rangle\right|,
$$

reduced to the number of lattice sites. With representation (112), we have

$$
M=\frac{1}{N}\left|\sum_{j}\left\langle t_{j x}^{\dagger} t_{j y}-t_{j y}^{\dagger} t_{j x}\right\rangle\right| .
$$

And representation (116) gives

$$
M=\frac{1}{N}\left|\sum_{j}\left\langle t_{j+}^{\dagger} t_{j+}-t_{j-}^{\dagger} t_{j-}\right\rangle\right| .
$$

Resorting to simplification (121) yields

$$
S_{1 j}^{x}=-S_{2 j}^{x}, \quad S_{1 j}^{y}=-S_{2 j}^{y}, \quad S_{1 j}^{z}=S_{2 j}^{z}, \quad S_{1 j}^{z}+S_{2 j}^{z}=-t_{j}^{\dagger} t_{j} .
$$

Finally, using normalization (123), we get

$$
M=\frac{1}{N} \sum_{j}\left\langle t_{j}^{\dagger} t_{j}\right\rangle=1-s^{2} .
$$

\subsection{Disordered phase}

The system Hamiltonian was initially a functional of the spin operators $\mathbf{S}_{1 j}$ and $\mathbf{S}_{2 j}$. With the bond-operator representation (106), it became a functional of the quasiparticle operators $s_{j}$ and $t_{j \alpha}$, with $\alpha=x, y, z$. After the canonical transformation (113), the Hamiltonian became a functional of $s_{j}$ and $t_{j \nu}$, where $\nu=+,-, 0$. With the following simplifications, resulting in Eqs. (121), the Hamiltonian acquired the form of a functional of $s$ and $t_{j}$. The sequence of these transformations can be symbolically depicted as

$$
\hat{H}\left[\mathbf{S}_{1 j}, \mathbf{S}_{2 j}\right] \longrightarrow \hat{H}\left[s_{j}, t_{j \alpha}\right] \longrightarrow \hat{H}\left[s_{j}, t_{j \nu}\right] \longrightarrow \hat{H}\left[s, t_{j}\right] .
$$

In the phase, where triplons are not condensed, one has

$$
\left\langle t_{j}\right\rangle=0 .
$$

To guarantee the normalization condition (122), one has to define the grand Hamiltonian

$$
H=\hat{H}\left[s, t_{j}\right]-\mu\left(N s^{2}+\sum_{j} t_{j}^{\dagger} t_{j}\right)
$$


with the Lagrange multiplier $\mu$ being the system chemical potential. The number of singletons is defined by minimizing the system thermodynamic potential, which leads to

$$
\left\langle\frac{\partial H}{\partial s}\right\rangle=0 .
$$

This is a necessary condition for the system stability.

\subsection{Triplon condensation}

The Bose-Einstein condensation of triplons implies the appearance of the order parameter

$$
\eta=\left\langle t_{j}\right\rangle \neq 0
$$

which does not depend on the site index due to the lattice periodicity. In order to correctly describe the condensed phase, we introduce the Bogolubov shift

$$
t_{j}=\eta+c_{j}
$$

Thus, the Hamiltonian now is a functional of $s, \eta, c_{j}$, which means the Hamiltonian transformation

$$
\hat{H}\left[s, t_{j}\right] \longrightarrow \hat{H}\left[s, \eta, c_{j}\right]
$$

The following numbers of quasiparticles are defined: the number of singletons

$$
N_{s}=\sum_{j} s^{2}=N s^{2},
$$

the number of condensed triplons

$$
N_{0}=\sum_{j}|\eta|^{2}=N \eta^{2}
$$

and the number of uncondensed triplons

$$
N_{1}=\sum_{j}\left\langle c_{j}^{\dagger} c_{j}\right\rangle=\left\langle\hat{N}_{1}\right\rangle,
$$

where the number operator of uncondensed triplons is

$$
\hat{N}_{1} \equiv \sum_{j} c_{j}^{\dagger} c_{j}
$$

The total number of quasiparticles, according to Eq. (123), is conserved:

$$
N_{s}+N_{0}+N_{1}=N
$$

To take into account these normalization conditions (133) to (135), it is necessary to introduce the grand Hamiltonian with the required number of Lagrange multipliers, which 
defines a representative ensemble $[16,18,145]$. Following the general approach, as applied to Bose-condensed systems [64-68], we need to introduce the grand Hamiltonian

$$
H=\hat{H}\left[s, \eta, c_{j}\right]-\mu_{s} N_{s}-\mu_{0} N_{0}-\mu_{1} \hat{N}_{1}
$$

The Lagrange multipliers $\mu_{s}, \mu_{0}$, and $\mu_{1}$ guarantee the validity of the normalization conditions (133), (134), and (135), playing the role of partial chemical potentials. The system chemical potential is defined by the variation of the system free energy $F$, which gives

$$
\mu=\frac{\partial F}{\partial N}=\frac{\partial F}{\partial N_{s}} \frac{\partial N_{s}}{\partial N}+\frac{\partial F}{\partial N_{0}} \frac{\partial N_{0}}{\partial N}+\frac{\partial F}{\partial N_{1}} \frac{\partial N_{1}}{\partial N} .
$$

This, with the partial derivatives

$$
\mu_{s}=\frac{\partial F}{\partial N_{s}}, \quad \mu_{0}=\frac{\partial F}{\partial N_{0}}, \quad \mu_{1}=\frac{\partial F}{\partial N_{1}}
$$

yields the system chemical potential

$$
\mu=\mu_{s} n_{s}+\mu_{0} n_{0}+\mu_{1} n_{1}
$$

in which the quasiparticle fractions are defined as

$$
n_{s}=\frac{\partial N_{s}}{\partial N}, \quad n_{0}=\frac{\partial N_{0}}{\partial N}, \quad n_{1}=\frac{\partial N_{1}}{\partial N}
$$

Not all partial Lagrange multipliers $\mu_{s}, \mu_{0}$, and $\mu_{1}$ are independent. Their relation follows from the condition of stability $\delta F=0$, which means

$$
\frac{\partial F}{\partial N_{s}} \delta N_{s}+\frac{\partial F}{\partial N_{0}} \delta N_{0}+\frac{\partial F}{\partial N_{1}} \delta N_{1}=0
$$

In view of Eqs. (139), this is equivalent to

$$
\mu_{s} \delta N_{s}+\mu_{0} \delta N_{0}+\mu_{1} \delta N_{1}=0 .
$$

Because of normalization (136), one has

$$
\delta N_{s}+\delta N_{0}+\delta N_{1}=0 .
$$

Using this, Eq. (142) reduces to

$$
\left(\mu_{0}-\mu_{s}\right) \delta N_{0}+\left(\mu_{1}-\mu_{s}\right) \delta N_{1}=0
$$

and further to

$$
\left(\mu_{0}-\mu_{s}\right) n_{0}+\left(\mu_{1}-\mu_{s}\right) n_{1}=0 .
$$

The latter gives

$$
\mu_{s}=\frac{\mu_{0} n_{0}+\mu_{1} n_{1}}{n_{0}+n_{1}} .
$$

Substituting this $\mu_{s}$ into Eq. (140) results in

$$
\mu=\mu_{s} .
$$


The quasiparticle fractions

$$
n_{s}=\frac{N_{s}}{N}=s^{2}, \quad n_{0}=\frac{N_{0}}{N}, \quad n_{1}=\frac{1}{N} \sum_{j}\left\langle c_{j}^{\dagger} c_{j}\right\rangle
$$

satisfy the normalization

$$
n_{s}+n_{0}+n_{1}=1 .
$$

Hence, the fraction of condensed triplons is given by the equality

$$
n_{0}=1-n_{1}-s^{2} .
$$

Magnetization (125) is

$$
M=1-s^{2}=n_{0}+n_{1} .
$$

The stability conditions, requiring the minimization of the thermodynamic potential, lead to the equations

$$
\left\langle\frac{\partial H}{\partial n_{s}}\right\rangle=0, \quad\left\langle\frac{\partial H}{\partial n_{0}}\right\rangle=0
$$

where the multipliers $\mu_{s}, \mu_{0}, \mu_{1}$ are fixed. The first of these equations is equivalent to Eq. (129). Finally, the chemical potential $\mu_{1}$ is defined by the condition of the triplon condensate existence $[16,18]$, which is equivalent to the requirement of a gapless spectrum, following from the Bogolubov [62] and Hugenholtz-Pines [69] theorems.

In this way, all quantities are uniquely defined. It is worth stressing the necessity of taking account of anomalous averages $\left\langle t_{j} t_{j}\right\rangle$. Neglecting these averages is mathematically unjustified, distorts thermodynamic relations, and spoils the phase transition order, making the Bose-Einstein condensation a first-order transition, as has been explained in Refs. $[16,18,67,146]$. While the proper account of the anomalous averages preserves the correct second order of the phase transition for Bose atoms $[16,18,147,148]$, as well as for triplons $[149,150]$.

Another principal point is the necessity of employing the representative ensemble with the grand Hamiltonian (137) in order to uniquely define a stable Bose-condensed system. For this, one has to introduce in the Hamiltonian the terms with the appropriate Lagrange multipliers. Forgetting some of these terms makes the system unstable. The majority of articles on triplon condensation do not use the correct grand Hamiltonian, hence, consider unstable systems.

Let us emphasize that condition (129) fixes the number of singletons. As far as the total number of all quasiparticles $N$ has been fixed from the beginning, being just the number of lattice sites, the number of triplons is also fixed. Thence, triplons are conserved quasiparticles and can condense. Being conserved quasiparticles, triplons are principally different from unconserved magnons. The latter cannot experience Bose condensation in an equilibrium system.

It is also important to remember that triplons are auxiliary quasiparticles that, strictly speaking, are not precisely defined, since in the bond-operator representation (106), they can be considered either as bosons or as fermions. Choosing them as bosons is an arbitrary decision. They could equivalently be chosen as fermions. So, the introduction of bosonic triplons is just a mathematical trick convenient for calculations, but the physical nature of these triplons as bosons is rather ambiguous. Contrary to this, the definition of 
magnons through the Holstein-Primakoff representation (87) uniquely prescribes to magnons the Bose-Einstein statistics. Magnons are bosons by their birth. And magnons describe the straightforward physical reality characterizing spin oscillations.

Unfortunately, many authors confuse triplons and magnons and write about magnon condensation in equilibrium dimer lattices. Of course, such a terminology, as has been stressed by Mills [36], is basically wrong. Triplons have nothing to do with magnons. The former are conserved quasiparticles and can condense in equilibrium, while the latter are not conserved and can never display equilibrium condensation.

\section{Strongly nonequilibrium condensates}

Unconserved quasiparticles cannot condense in equilibrium, although some of them can condense in nonequilibrium systems, e.g., excitons, polaritons, and photons. Coherent motion of transverse spins can also be interpreted as nonequilibrium magnon condensation. However, one should keep in mind that the Holstein-Primakoff transformation is not valid for strongly nonequilibrium spin systems, because of which it cannot not be used for the latter.

Nonequilibrium is favorable for the possibility of condensation of unconserved quasiparticle. But, contrary to this, nonequilibrium perturbations usually destroy the condensate of conserved particles. In this regard, it is illustrative to study what happens with the Bose system, prepared in the Bose-condensed state, after the system is subject to the action of increasing external perturbation driving the system far from its equilibrium state.

\subsection{Coherent topological modes}

Trapped atoms provide a very convenient object for studying well controlled transformations of an equilibrium Bose-condensed system into a highly excited state. There are different ways of exciting a Bose-condensed system by external fields. All of these ways can be mathematically classified into two categories. One way is to act on the atomic density by applying an external field that is characterized by a time-dependent potential $V(\mathbf{r}, t)$. Another method is to make the atomic scattering length $a_{s}(t)$ a function of time by invoking the Feshbach resonance techniques. Generally, the scattering length can be made a function of time and space. These two methods lead to similar consequences.

When the amplitude of the perturbing field is small, elementary collective excitations are produced, representing density fluctuations around the given equilibrium state. For short wavelengths $\lambda$, much shorter that the effective system size $L$, collective excitations in a nonuniform system are described by the Bogolubov spectrum

$$
\varepsilon(\mathbf{k}, \mathbf{r})=\sqrt{c^{2}(\mathbf{r}) k^{2}+\left(\frac{k^{2}}{2 m}\right)^{2}},
$$

where $c(\mathbf{r})$ is local sound velocity. For long waves, such that $\lambda \gg L$, the spectrum of collective excitations is discrete and depends on the trap geometry [1-12]. These elementary collective excitations do not change the condensate fraction.

But even rather weak perturbations may drive the system far from equilibrium, if the external field alternates with a frequency that is in resonance with a transition frequency between two energy levels of the trapped system. Recall that, for a trapped system, the 
spectrum of the stationary Gross-Pitaevskii equation is discrete and, because of atomic interactions, it is not equidistant. This allows one to tune the frequency of the external alternating field close to one of the transition frequencies.

Coherent topological modes are the solutions of the stationary condensate-function equation $[16,18,43,53,54]$. At temperatures close to zero and asymptotically weak interactions, the condensate-function equation reduces to the stationary Gross-Pitaevskii equation

$$
\hat{H}\left[\varphi_{n}\right] \varphi_{n}(\mathbf{r})=E_{n} \varphi_{n}(\mathbf{r}),
$$

with the Gross-Pitaevskii Hamiltonian

$$
\hat{H}[\varphi]=-\frac{\nabla^{2}}{2 m}+U(\mathbf{r})+N_{0} \Phi_{0}|\varphi|^{2},
$$

in which $U(\mathbf{r})$ is a trapping potential, $N_{0}$ is the number of condensed atoms, and

$$
\Phi_{0} \equiv 4 \pi \frac{a_{s}}{m}
$$

is interaction strength, with $a_{s}$ being scattering length. The modes are called coherent, since they correspond to the Bose-condensed part of the system, which, by definition, is coherent. And they are termed topological because the corresponding atomic density is topologically different from the density of the ground state.

Suppose that, in addition to the trapping potential, the system is subject to the action of an alternating external potential

$$
V(\mathbf{r}, t)=V_{1}(\mathbf{r}) \cos (\omega t)+V_{2}(\mathbf{r}) \sin (\omega t),
$$

whose frequency is tuned close to a chosen transition frequency

$$
\omega_{21} \equiv E_{2}-E_{1} .
$$

This implies that the resonance condition

$$
\left|\frac{\Delta \omega}{\omega_{21}}\right| \ll 1 \quad\left(\Delta \omega \equiv \omega-\omega_{21}\right)
$$

is valid. The problem is analogous to that of resonance transitions of atoms, with the principal difference in its nonlinearity caused by atomic interactions.

Dynamics of the system is described by the time-dependent condensate-function equation $[16,18,53]$ or, at zero temperature and weak interactions, by the Gross-Pitaevskii equation that is nothing but a nonlinear Schrödinger equation

$$
i \frac{\partial}{\partial t} \varphi(\mathbf{r}, t)=\{\hat{H}[\varphi]+V(\mathbf{r}, t)\} \varphi(\mathbf{r}, t) .
$$

The solution to this equation can be represented in the form of the expansion over the coherent modes,

$$
\varphi(\mathbf{r}, t)=\sum_{n} c_{n}(t) \varphi_{n}(\mathbf{r}) e^{-i E_{n} t} .
$$


The fractional mode populations are given by the expressions

$$
p_{n}(t) \equiv\left|c_{n}(t)\right|^{2} .
$$

Under the resonance condition (155), it is feasible to excite the coherent modes employing rather weak external fields. Similarly, by several alternating fields, one can generate several coherent modes [51,52]. If we increase the amplitude of the perturbing external fields, then the topological coherent modes, in addition to the direct resonance, with $\omega=\omega_{21}$, can also be generated by other processes, such as harmonic generation under condition

$$
n \omega=\omega_{21} \quad(n=2,3, \ldots),
$$

parametric conversion, when

$$
\omega_{1} \pm \omega_{2}=\omega_{21}
$$

and other combined resonances, when

$$
\sum_{i} n_{i} \omega_{i}=\omega_{21} \quad\left(n_{i}= \pm 1, \pm 2, \ldots\right) .
$$

Thus, the increasing perturbation can generate a variety of different coherent modes. And, with a sufficiently strong perturbation, strict resonance conditions are not required.

Among all these modes, there are quantized vortices. Their generation in a trap, perturbed by external fields, can be done so as to produce vortices with a chosen circulation, or the pairs of vortices and antivortices can be generated. One often employs cylindrical traps characterized by a transverse frequency $\omega_{\perp}$ and longitudinal frequency $\omega_{z}$, with the aspect ratio

$$
\alpha \equiv \frac{\omega_{z}}{\omega_{\perp}}=\left(\frac{l_{\perp}}{l_{z}}\right)^{2},
$$

where the related oscillator lengths are

$$
l_{\perp} \equiv \frac{1}{\sqrt{m \omega_{\perp}}}, \quad l_{z} \equiv \frac{1}{\sqrt{m \omega_{z}}} .
$$

The strength of atomic interactions can be described by the dimensionless coupling parameter

$$
g \equiv 4 \pi N \frac{a_{s}}{l_{\perp}} .
$$

The basic vortex, having unit circulation [4], possesses the energy

$$
\omega_{v o r}=\frac{5}{2 m R_{T F}^{2}} \ln \left(0.7 \frac{R_{T F}}{\xi}\right),
$$

where the notation is used for the Thomas-Fermi radius squared

$$
R_{T F}^{2} \equiv \frac{1}{m \omega_{\perp}}\left(\frac{15}{4 \pi} \alpha g\right)^{2 / 5}
$$

and the healing length

$$
\xi \equiv \frac{1}{\sqrt{2 m \rho \Phi_{0}}} .
$$


The vortex energy can be written in the form

$$
\omega_{\text {vor }}=\frac{0.9 \omega_{\perp}}{(\alpha g)^{2 / 5}} \ln (0.8 \alpha g) .
$$

The number of atoms $N$ in a trap is usually so large that the coupling parameter (163) is a large number $g \gg 1$. Then the dependence of the coherent-mode transition frequencies on the coupling parameter is such that

$$
\omega_{m n} \propto(\alpha g)^{2 / 5},
$$

hence, these energies increase with $g$. While the energy of the basic vortex (165) decreases with $g$. This is why the basic vortex is the most energetically stable among all coherent modes, so that rather intensive perturbation creates mainly such basic vortices $[5,18]$. The core of a vortex is the region with condensate depletion. Thence, vortex generation depletes the condensate fraction.

\subsection{Creation of separate vortices}

When the strength of external perturbations continues growing, this pumps into the system additional energy. The action of an alternating potential $V(\mathbf{r}, t)$ increases the system energy so that the injected energy per atom is

$$
E_{i n j}=\frac{1}{N} \int \rho(\mathbf{r}, t)\left|\frac{\partial V(\mathbf{r}, t)}{\partial t}\right| d \mathbf{r} d t .
$$

Let the pumping potential have the amplitude $A$ and frequency $\omega$. Then the injected energy (166), during the time interval $\left[t, t^{\prime}\right]$, approximately is

$$
E_{i n j} \approx A \omega\left(t-t^{\prime}\right) .
$$

A single vortex is created when the injected energy is of the order of the vortex energy, $E_{i n j} \sim \omega_{\text {vor }}$, which implies

$$
A \omega\left(t-t_{0}\right) \sim \omega_{v o r} .
$$

Therefore the crossover line of a single vortex creation, on the amplitude-time plane, is given by the expression

$$
A_{\text {vor }} \sim \frac{\omega_{\text {vor }}}{\omega\left(t-t_{0}\right)} .
$$

Several vortices, of the number $N_{v o r}$, can be generated when the injected energy reaches the value $E_{\text {inj }} \sim N_{\text {vor }} \omega_{\text {vor }}$, hence when

$$
A \omega\left(t-t_{1}\right) \sim N_{\text {vor }} \omega_{\text {vor }} .
$$

The more vortices are produced, the more condensate fraction is depleted, though the system remains yet superfluid. 


\subsection{Regime of quantum turbulence}

Producing more and more vortices (and antivortices), one can reach the state where the vortices form a random tangle. Such a random tangle of vortices signifies the appearance of the state of quantum turbulence [151-158]. The number of vortices, when the random tangle is being formed can be estimated [18] as

$$
N_{v o r} \sim \frac{l_{0}}{\xi}
$$

where the effective trap parameters are

$$
l_{0} \equiv \frac{1}{\sqrt{m \omega_{0}}}=\left(l_{\perp}^{2} l_{z}\right)^{1 / 3}, \quad \omega_{0}=\frac{1}{m l_{0}^{2}}=\left(\omega_{\perp}^{2} \omega_{z}\right)^{1 / 3} .
$$

This defines the crossover line of the starting turbulence

$$
A_{\text {tur }} \sim \frac{l_{0} \omega_{\text {vor }}}{\xi \omega\left(t-t_{1}\right)}
$$

In the turbulent regime the condensate fraction is getting more and more depleted. But the system can yet be treated as superfluid.

\subsection{Spatial condensate granulation}

With the developed turbulence, the condensate fraction becomes small. And finally, the condensate breaks into spatially separated pieces, or grains, that are immersed into the normal phase without condensate. Then locally, in each grain, there is condensate. However, since the grains are spatially separated, superfluidity through the whole volume cannot arise. Generally, such a state is called heterophase, as far as it is formed by a mixture of different phases, randomly distributed in space [145,159-162]. In the present case, it can be termed granulated condensate [163]. The typical size of grains with condensate is given by the localization length

$$
l_{l o c}=\frac{1}{m^{2} E_{i n j}^{2} l_{0}^{3}}=\left(\frac{\omega_{0}}{E_{i n j}}\right)^{2} l_{0} .
$$

These grains are mesoscopic in size, in the sense that the above localization length is larger than the mean interatomic distance $a$, but shorter than the effective trap size:

$$
a<l_{l o c}<l_{0}
$$

The granulation starts when the injected energy is so large that the localization length becomes of the order of the trap size,

$$
l_{l o c} \sim l_{0}, \quad E_{i n j} \sim \omega_{0} .
$$

This defines the crossover line of granulation

$$
A_{h e t} \sim \frac{\omega_{0}}{\omega\left(t-t_{2}\right)}
$$


Increasing the injected energy diminishes the grain size (171). And the condensate is completely destroyed, when the localization length (171) reduces to the order of the mean interatomic distance:

$$
l_{l o c} \sim a, \quad E_{i n j} \sim \omega_{0} \sqrt{\frac{l_{0}}{a}}
$$

The corresponding crossover line

$$
A_{\text {nor }} \sim \frac{\omega_{0}}{\omega\left(t-t_{3}\right)} \sqrt{\frac{l_{0}}{a}}
$$

shows where the granulated condensate is destroyed and the atomic cloud becomes a normal system without condensate. Since this system is strongly nonequilibrium, it can be named chaotic fluid.

\subsection{Amplitude-time phase diagram}

The above discussion can be summarized with the phase diagram in Fig. 1 on the plane of the pumping field amplitude versus pumping time. The sequence of these states, except the chaotic fluid, has been observed in experiments [164-167]. The state of chaotic fluid has not yet been reached. The details for the phase diagram can be found in Refs. [166,167].

In experiments [164-167], trapped atoms of ${ }^{8} 7 \mathrm{Rb}$ have been treated, having mass $m=$ $1.445 \times 10^{-22} \mathrm{~g}$ and scattering length $a_{s}=0.577 \times 10^{-6} \mathrm{~cm}$. The cloud was cooled down to low temperatures, much lower that the critical temperature of condensation $T_{c}=276 \mathrm{nK}$, so that almost all $N=2 \times 10^{5}$ atoms were condensed. A cylindrical trap was used with the trap characteristics

$$
\begin{gathered}
\omega_{\perp}=2 \pi \times 210 \mathrm{~Hz}=1.32 \times 10^{3} \mathrm{~s}^{-1}, \quad \omega_{z}=2 \pi \times 23 \mathrm{~Hz}=1.45 \times 10^{2} \mathrm{~s}^{-1}, \quad \omega_{0}=0.63 \times 10^{3} \mathrm{~s}^{-1}, \\
l_{\perp}=0.74 \times 10^{-4} \mathrm{~cm}, \quad l_{z}=2.25 \times 10^{-4} \mathrm{~cm}, \quad l_{0}=1.08 \times 10^{-4} \mathrm{~cm} .
\end{gathered}
$$

This is an elongated trap with the aspect ratio $\alpha \equiv \omega_{z} / \omega_{\perp}=0.11$.

The effective atomic volume $V_{\text {eff }}$, average density $\rho$, mean interatomic distance $a$, and gas parameter $\gamma$, respectively, are

$$
\begin{gathered}
V_{e f f} \equiv \pi l_{\perp}^{2} 2 l_{z}=2 \pi l_{0}^{3}=0.78 \times 10^{-11} \mathrm{~cm}^{3}, \quad \rho \sim 2.55 \times 10^{15} \mathrm{~cm}^{-3} \\
a \equiv \rho^{-1 / 3}=0.73 \times 10^{-5} \mathrm{~cm}, \quad \gamma \equiv \rho^{1 / 3} a_{s}=\frac{a_{s}}{a}=0.079 .
\end{gathered}
$$

The dimensionless coupling parameters are

$$
g=4 \pi N \frac{a_{s}}{l_{\perp}}=1.96 \times 10^{4}, \quad \alpha g=2.16 \times 10^{3}
$$

which implies strong effective atomic interactions.

The trapped system of condensed atoms was subject to the action of a modulating field of frequency

$$
\omega=2 \pi \times 200 \mathrm{~Hz}=1.26 \times 10^{3} \mathrm{~s}^{-1},
$$

hence, with the modulation period $T_{\text {mod }} \equiv 2 \pi / \omega=5 \times 10^{-3}$ s. The action of this perturbation lasted during the modulation time $t_{\text {mod }}=0.02-0.06 \mathrm{~s}$. 
The time of local equilibration is

$$
t_{l o c}=\frac{m}{\hbar \rho a_{s}}=0.93 \times 10^{-4} \mathrm{~s} .
$$

Then the relation between the characteristic times is

$$
t_{l o c} \ll T_{\text {mod }} \ll t_{\text {mod }} .
$$

This means that the atomic system, during the process of modulation, has always been in the state of local equilibrium.

The healing length, defining the vortex core, is

$$
\xi \equiv \frac{1}{\sqrt{8 \pi \rho a_{s}}}=0.52 \times 10^{-5} \mathrm{~cm}
$$

Therefore the characteristic lengths are related as

$$
a_{s} \ll \xi \sim a \ll l_{0} \text {. }
$$

The vortex energy is $\omega_{\text {vor }}=0.41 \times 10^{3} \mathrm{~s}^{-1}$. The turbulent regime starts when the number of the generated vortices reaches the value

$$
N_{\text {vor }}=\frac{l_{0}}{\xi} \approx 20
$$

which is in good agreement with experiment [166,167].

The consideration of the present section illustrates how the system of conserved atoms, initially prepared in an almost pure Bose-condensed state, and being subject to the perturbation by external alternating fields, goes through a sequence of nonequilibrium regimes, ending with a normal chaotic fluid without condensate. The initial condensate fraction has been almost $100 \%$, while the final state contains no condensate. Nonequilibrium external perturbation depletes the condensate fraction of conserved particles.

A challenging problem would be to investigate experimentally the opposite process of equilibration of an excited atomic system, from the state of chaotic fluid back to the Bosecondensed state. Such problems of equilibration of finite quantum systems attract now high attention, as can be inferred form the review articles [168-170].

\section{Conclusion}

For the phenomenon of the Bose-Einstein condensation, the distinction between particles and quasiparticles is secondary. The most important is whether their number is conserved or not. Conserved particles and quasiparticles can experience Bose-Einstein condensation in equilibrium systems as well as can form nonequilibrium condensates. But unconserved quasiparticles cannot exhibit equilibrium condensation. However, they can display condensation in nonequilibrium states, when an external pumping creates a sufficient number of quasiparticles. Examples are nonequilibrium condensates of excitons, polaritons, and photons. 
It is possible to interpret the shape and Jahn-Teller phase transitions as condensation of phonons. However this interpretation is useful solely at the phase transition points, where the system is actually unstable. After the phase transition has occurred, there are no condensed phonons, but it is just necessary to redefine the mean atomic locations. It is possible to avoid talking on phonon condensation by interpreting shape transitions as a sharp variation of the mean atomic locations. Self-consistently defined phonons never condense, whether in equilibrium or nonequilibrium systems.

Among quasiparticles, there are those that enjoy direct physical meaning, such as phonons and magnons. And there are auxiliary quasiparticles, appearing in the process of mathematical transformations and having no precise physical meaning, for instance, Schwinger bosons, slave bosons, or singletons and triplons. These auxiliary quasiparticles even are not uniquely defined as bosons. If, by force, they are assumed to be bosons, they can display equilibrium condensation, provided they are conserved.

When considering quasiparticle condensation, it is principally important to employ a correct terminology, not confusing conserved with unconserved quasiparticles. For example, triplons are auxiliary conserved quasiparticles that are formally allowed to condense in equilibrium. And triplons must not be confused with magnons which are physical unconserved quasiparticles that cannot experience equilibrium condensation.

The appearance of average transverse magnetization in strongly nonequilibrium spin systems can be interpreted as magnon condensation, though the Holstein-Primakoff transformation for such nonequilibrium situations is not applicable.

To exhibit condensation, unconserved quasiparticles require nonequilibrium conditions. Contrary to this, the condensate of conserved particles becomes depleted by nonequilibrium external perturbations.

\section{Acknowledgement}

I am grateful for discussions to V.S. Bagnato, A. Garbaly, and E.P. Yukalova. Financial support from the Russian Foundation for Basic Research is appreciated. 


\section{References}

[1] L. Pitaevskii and S. Stringari, Bose-Einstein Condensation (Clarendon, Oxford, 2003).

[2] E.H. Lieb, R. Seiringer, J.P. Solovej, and J. Yngvason, The Mathematics of the Bose Gas and Its Condensation (Birkhauser, Basel, 2005).

[3] V. Letokhov, Laser Control of Atoms and Molecules (Oxford University, New York, 2007).

[4] C.J. Pethik and H. Smith, Bose-Einstein Condensation in Dilute Gases (Cambridge University, Cambridge, 2008).

[5] P.W. Courteille, V.S. Bagnato, and V.I. Yukalov, Laser Phys. 11, 659 (2001).

[6] J.O. Andersen, Rev. Mod. Phys. 76, 599 (2004).

[7] V.I. Yukalov, Laser Phys. Lett. 1, 435 (2004).

[8] K. Bongs and K. Sengstock, Rep. Prog. Phys. 67, 907 (2004).

[9] V.I. Yukalov and M.D. Girardeau, Laser Phys. Lett. 2, 375 (2005).

[10] A. Posazhennikova, Rev. Mod. Phys. 78, 1111 (2006).

[11] V.I. Yukalov, Laser Phys. Lett. 4, 632 (2007).

[12] N.P. Proukakis and B. Jackson, J. Phys. B 41, 203002 (2008).

[13] V.A. Yurovsky, M. Olshanii, and D.S. Weiss, Adv. At. Mol. Opt. Phys. 55, 61 (2008).

[14] W. Ketterle, and M.W. Zwierlein, Riv. Nuovo Cimento 31, 247 (2008).

[15] C. Moseley, O. Fialko, and K. Ziegler, Ann. Phys. (Berlin) 17, 561 (2008).

[16] V.I. Yukalov, Laser Phys. 19, 1 (2009).

[17] A.L. Fetter, Rev. Mod. Phys. 81, 647 (2009).

[18] V.I. Yukalov, Phys. Part. Nucl. 42, 460 (2011).

[19] J.M. Blatt, K.W. Böer, and W. Brandt, Phys. Rev. 126, 1691 (1962).

[20] L. Keldysh and A.N. Kozlov, JETP Lett. 27, 521 (1968).

[21] W. Kohn and D. Sherrington, Rev. Mod. Phys. 42, 1 (1970).

[22] L.V. Butov, Solid State Commun. 127, 89 (2003).

[23] J.P. Eisenstein and A.H. MacDonald, Nature 432, 691 (2004).

[24] H. Deng, G. Weihs, C. Santory, J. Bloch, and Y. Yamamoto, Science 298, 199 (2002). 
[25] J. Keeling, F.M. Marchetti, M.H. Szymanska, and P.B. Littlewood, Semicond. Sci. Technol. 22, 1 (2007).

[26] M.H. Szymanska, J. Keeling, and P.B. Littlewood, Phys. Rev. B 75, 195331 (2007).

[27] E.R. Bittner and C. Silva, arXiv:1104.2017 (2011).

[28] J. Klaers, J. Schmitt, F. Vewinger, and M. Weitz, Nature 468, 545 (2010).

[29] T. Nikuni, M. Oshikawa, A. Oosawa, and H. Tanaka, Phys. Rev. Lett. 84, 5868 (2000).

[30] M. Matsumoto, B. Normand, T.M. Rice, and M. Sigrist, Phys. Rev. B 69, 054423 (2004).

[31] G. Misguich and M. Oshikawa, J. Phys. Soc. Jpn. 73, 3429 (2004).

[32] J. Sirker, A. Weisse, and O.P. Sushkov, J. Phys. Soc. Jpn. 74, 129 (2005).

[33] T. Matsubara and H. Matsuda, Prog. Theor. Phys. 16, 569 (1956).

[34] E.G. Batyev and L.S. Braginskii, J. Exp. Theor. Phys. 60, 781 (1984).

[35] Y. Kagan and L.A. Manakova, Phys. Lett. A 361, 401 (2007).

[36] D.L. Mills, Phys. Rev. Lett. 98, 039701 (2007).

[37] R.P. Feynman and S. Weinberg, Elementary Particles and the Laws of Physics (Cambridge University, Cambridge, 1987).

[38] N. Manton and P. Sutcliffe, (2004). Topological solitons (Cambridge University, Cambridge, 2004).

[39] Y.V. Kartashov, B.A. Malomed, and L. Torner, Rev. Mod. Phys. 83, 247 (2011).

[40] A.M. Kosevich, B.A. Ivanov, and A.S. Kovalev, Phys. Rep. 194, 117 (1990).

[41] S. Flach and S.R. Willis, Phys. Rep. 295, 181 (1998).

[42] K.P. Marzlin and V.I. Yukalov, Eur. Phys. J. D 33, 253 (2005).

[43] V.I. Yukalov, E.P. Yukalova, and V.S. Bagnato, Phys. Rev. A 56, 4845 (1997).

[44] V.I. Yukalov, E.P. Yukalova, and V.S. Bagnato, Laser Phys. 10, 26 (2000).

[45] V.I. Yukalov, E.P. Yukalova, and V.S. Bagnato, Laser Phys. 11, 455 (2001).

[46] V.I. Yukalov, E.P. Yukalova, and V.S. Bagnato, Laser Phys. 12, 231 (2002).

[47] V.I. Yukalov, E.P. Yukalova, and V.S. Bagnato, Laser Phys. 12, 1325 (2002).

[48] V.I. Yukalov, E.P. Yukalova, and V.S. Bagnato, Phys. Rev. A 66, 043602 (2002).

[49] V.I. Yukalov, E.P. Yukalova, and V.S. Bagnato, Laser Phys. 13, 551 (2003). 
[50] V.I. Yukalov, E.P. Yukalova, and V.S. Bagnato, Laser Phys. 13, 861 (2003).

[51] V.I. Yukalov, K.P. Marzlin, and E.P. Yukalova, Laser Phys. 14, 565 (2004).

[52] V.I. Yukalov, K.P. Marzlin, and E.P. Yukalova, Phys. Rev. A 69, 023620 (2004).

[53] V.I. Yukalov, Laser Phys. Lett. 3, 406 (2006).

[54] V.I. Yukalov and V.S. Bagnato, Laser Phys. Lett 6, 399 (2009).

[55] R.L. Stratonovich, Phys. Dokl. 2, 416 (1958).

[56] A. Khare, Fractional Statistics and Quantum Theory (World Scientific, Singapore, 2005).

[57] O. Penrose and L. Onsager, Phys. Rev. 104, 576 (1956).

[58] A.J. Coleman and V.I. Yukalov, Mod. Phys. Lett. B 5, 1679 (1991).

[59] A.J. Coleman and V.I. Yukalov, Nuovo Cimento B 107, 535 (1992).

[60] A.J. Coleman and V.I. Yukalov, Nuovo Cimento B 108, 1377 (1993).

[61] N.N. Bogolubov, Lectures on Quantum Statistics (Gordon and Breach, New York, 1967), Vol. 1.

[62] N.N. Bogolubov, Lectures on Quantum Statistics (Gordon and Breach, New York, 1970), Vol. 2.

[63] V.I. Yukalov, Laser Phys. 16, 511 (2006).

[64] V.I. Yukalov, Phys. Rev. E 72, 066119 (2005).

[65] V.I. Yukalov, Phys. Lett. A 359, 712 (2006).

[66] V.I. Yukalov, Int. J. Mod. Phys. B 21, 69 (2007).

[67] V.I. Yukalov, Ann. Phys. (N.Y.) 323, 461 (2008).

[68] V.I. Yukalov, Phys. Lett. A 375, 2797 (2011).

[69] N.M. Hugenholtz and D. Pines, Phys. Rev. 116, 489 (1959).

[70] P.C. Hohenberg and P.C. Martin, Ann. Phys. (N.Y.) 34, 291 (1965).

[71] J. Gavoret and P. Noziéres, Ann. Phys. (N.Y.) 28, 349 (1964).

[72] V.I. Yukalov, Mod. Phys. Lett. B 5, 725 (1991).

[73] V. Kalogera and G. Baym, Astrophys. J. Lett. 470, 61 (1996).

[74] T.S. Olson, Phys. Rev. C 63, 015802 (2000).

[75] T.J. Battefeld and D.A. Easson, Phys. Rev. D 70, 103516 (2004). 
[76] T. Banks, W. Fischler, and L. Mannelli, Phys. Rev. D 71, 123514 (2005).

[77] V.I. Yukalov, Laser Phys. Lett. 7, 831 (2010).

[78] V.I. Yukalov, Phys. Rev. A 72, 033608 (2005).

[79] A.A. High, J.R. Leonard, A.T. Hammack, M.M. Fogler, L.V. Butov, A.V. Kavokin, K.L. Campmann, and A.C. Gossard, arXiv:1109.0253 (2011).

[80] O. Morsch and M. Oberthaler, Rev. Mod. Phys. 78, 179 (2006).

[81] I. Bloch, J. Dalibard, and W. Zwerger, Rev. Mod. Phys. 80, 885 (2008).

[82] J. Sebby-Strabley, M. Anderlini, P.S. Jessen, and J.V. Porto, Phys. Rev. A 73, 033605 (2006).

[83] J. Sebby-Strabley, B.L. Brown, M. Anderlini, P.J. Lee, W.D. Phillips, J.V. Porto, and P.R. Johnson, Phys. Rev. Lett. 98, 200405 (2007).

[84] P.J. Lee, M. Anderlini, B.L. Brown, J. Sebby-Strabley, W.D. Phillips, and J.V. Porto, Phys. Rev. Lett. 99, 020402 (2007).

[85] S. Fölling, S. Trotzky, P. Cheinet, M. Feld, R. Saers, A. Widera, T. Müller, and I. Bloch, Nature 448, 1029 (2007).

[86] P. Cheinet, S. Trotzky, M. Feld, U. Schnorrberger, M. Moreno-Cardoner, S. Fölling, and I. Bloch, Phys. Rev. Lett. 101, 090404 (2008).

[87] I. Danshita, J.E. Williams, C.A.R. Sa de Melo, and C.W. Clark, Phys. Rev. A 76, 043606 (2007).

[88] I. Danshita, C.A.R. Sa de Melo, and C.W. Clark, Phys. Rev. A 77, 063609 (2008).

[89] V.I. Yukalov and E.P. Yukalova, Phys. Rev. A 78, 063610 (2008).

[90] V.I. Yukalov and E.P. Yukalova, Laser Phys. Lett. 6, 235 (2009).

[91] V.I. Yukalov and E.P. Yukalova, Phys. Lett. A 373, 1301 (2009).

[92] V.I. Yukalov and E.P. Yukalova, Laser Phys. 21, 1448 (2011).

[93] V.O. Nesterenko, A.N. Novikov, F.F. de Souza Cruz, and E.L. Lapolli, Laser Phys. 19, $616(2009)$.

[94] C. Weiss and N. Teichmann, Laser Phys. 19, 673 (2009).

[95] B. Oleś, P. Ziń, J. Chwedeńczuk, K. Sacha, and M. Trippenbach, Laser Phys. 20, 671 (2010).

[96] V.I. Yukalov, A. Rakhimov, and S. Mardonov, Laser Phys. 21, 264 (2011).

[97] V.I. Yukalov, Symmetry 2, 40 (2010). 
[98] V.I. Yukalov, Physica A 155, 519 (1989).

[99] H.A. Jahn and E. Teller, Proc. Roy. Soc. London A 161, 220 (1937).

[100] I.B. Bersuker, The Jahn-Teller Effect (Cambridge University, Cambridge, 2006).

[101] T. Holstein and H. Primakoff, Phys. Rev. 58, 1098 (1940).

[102] S.V. Tyablikov, Methods in Quantum Theory of Magnetism (Plenum, New York, 1967).

[103] A.S. Borovik-Romanov, Y.M. Bunkov, V.V. Dmitriev, and Y.M. Mukharsky, JETP Lett. 40, 1033 (1984).

[104] S.O. Demokritov, V.E. Demidov, O. Dzyapko, G.A. Melkov, A.A. Serga, B. Hillebrands, and A.N. Slavin, Nature 443, 430 (2006).

[105] V.E. Demidov, O. Dzyapko, S.O. Demokritov, G.A. Melkov, and A.N. Slavin, Phys. Rev. Lett. 100, 047205 (2008).

[106] G.E. Volovik, J. Low Temp. Phys. 153, 266 (2008).

[107] Y.M. Bunkov, Phys. Usp. 53, 848 (2010).

[108] Y.M. Bunkov and G.E. Volovik, J. Phys. Condens. Matter 22, 164210 (2010).

[109] V.I. Yukalov, Phys. Rev. Lett. 75, 3000 (1995).

[110] V.I. Yukalov, Laser Phys. 5, 526 (1995).

[111] V.I. Yukalov, Laser Phys. 5, 970 (1995).

[112] V.I. Yukalov, Phys. Rev. B 53, 9232 (1996).

[113] V.I. Yukalov and E.P. Yukalova, Phys. Part. Nucl. 31, 561 (2000).

[114] V.I. Yukalov and E.P. Yukalova, Phys. Rev. Lett. 88, 257601 (2002).

[115] V.I. Yukalov, Laser Phys. 12, 1089 (2002).

[116] V.I. Yukalov and E.P. Yukalova, Phys. Part. Nucl. 35, 348 (2004).

[117] V.I. Yukalov, Phys. Rev. B 71, 184432 (2005).

[118] V.I. Yukalov, V.K. Henner, and P.V. Kharebov, Phys. Rev. B 77, 134427 (2008).

[119] V.I. Yukalov, V.K. Henner, P.V. Kharebov, and E.P. Yukalova, Laser Phys. Lett. 5, 887 (2008).

[120] V.I. Yukalov and E.P. Yukalova, Laser Phys. Lett. 8, 804 (2011).

[121] A.V. Chubukov and D.K. Morr, Phys. Rev. B 52, 3521 (1995).

[122] A.V. Syromyatnikov, Phys. Rev. B 75, 134421 (2007). 
[123] V.I. Yukalov and A.S. Shumovsky, Lectures on Phase Transitions (World Scientific, Singapore, 1990).

[124] T. Giarmachi and A.M. Tsvelik, Phys. Rev. B 59, 11398 (1999).

[125] S. Wessel and S. Haas, Phys. Rev. B 62, 316 (2000).

[126] P. Jordan and E. Wigner, Z. Phys. 47, 631 (1928).

[127] N.N. Bogolubov, Lectures on Quantum Statistics (Ryadyanska Shkola, Kiev, 1949).

[128] J. Schwinger, On Angular Momentum (US Atomic Energy Commission, Washington, 1952).

[129] A. Auerbach, Interacting Electrons and Quantum Magnetism (Springer, New York, 1994).

[130] A.J. Coleman, E.P. Yukalova, and V.I. Yukalov, Int. J. Quantum Chem. 54, 211 (1995).

[131] G. Su and M. Suzuki, Int. J. Mod. Phys. B 13, 925 (1999).

[132] S. Sarker, C. Jayaprakash, H.R. Krishnamurthy, and M. Ma, Phys. Rev. B 40, 5028 (1989).

[133] D. Yoshioka, J. Phys. Soc. Jpn. 58, 3733 (1989).

[134] T.N. De Silva, M. Ma, and F.C. Zhang, Phys. Rev. B 66, 104417 (2002).

[135] S.E. Barnes, J. Phys. F 6, 1375 (1976).

[136] N. Read and D.M. Newns, J. Phys. C 16, 3273 (1983).

[137] N. Read and D.M. Newns, Adv. Phys. 36, 799 (1988).

[138] P.S. Riseborough, Phys. Rev. B 45, 13984 (1992).

[139] S.H.S. Salk and S.S. Lee, Physica B 284, 441 (2000).

[140] D.P. Arovas and A. Auerbach, Phys. Rev. B 38, 316 (1988).

[141] D. Yoshioka, J. Phys. Soc. Jpn. 58, 1516 (1989).

[142] J.W. Rasul and Y. Bai, J. Phys. Condens. Matter 8, 4495 (1996).

[143] E.A. Kochetov and A. Ferraz, Phys. Rev. B 70, 052508 (2004).

[144] S. Sachdev and R.N. Bhatt, Phys. Rev. B 41, 9323 (1990).

[145] V.I. Yukalov, Phys. Rep. 208, 395 (1991).

[146] V.I. Yukalov and E.P. Yukalova, Laser Phys. Lett. 2, 506 (2005).

[147] V.I. Yukalov and E.P. Yukalova, Phys. Rev. A 74, 063623 (2006). 
[148] V.I. Yukalov and E.P. Yukalova, Phys. Rev. A 76, 013602 (2007).

[149] A. Rakhimov, E.Y. Sherman, and C.K. Kim, Phys. Rev. B 81, 020407 (2010).

[150] A. Rakhimov, S. Mardonov, and E.Y. Sherman, Ann. Phys. (N.Y.) 326, 2499 (2011).

[151] E.B. Sonin, Rev. Mod. Phys. 59, 87 (1987).

[152] B.V. Svistunov, Phys. Rev. B 52, 3647 (1995).

[153] S.K. Nemirovskii, W. Fiszdon, Rev. Mod. Phys. 67, (1995) 37 (1995).

[154] W.F. Vinen, J.J. Niemela, J. Low Temp. Phys. 128, 167 (2002).

[155] W.F. Vinen, J. Low Temp. Phys. 145, 7 (2006).

[156] M. Tsubota, J. Phys. Soc. Jpn. 77, (2008) 111006 (2008).

[157] V.I. Yukalov, Laser Phys. Lett. 7, 467 (2010).

[158] M. Tsubota, K. Kasamatsu, M. Kobayashi, arXiv:1004.5458 (2010).

[159] V.I. Yukalov, Theor. Math. Phys. 26, 274 (1976).

[160] V.I. Yukalov, Theor. Math. Phys. 28, 652 (1976).

[161] V.I. Yukalov, Phys. Lett. A 81, 249 (1981).

[162] V.I. Yukalov, Phys. Lett. A 81, 433 (1981).

[163] V.I. Yukalov, E.P. Yukalova, and V.S. Bagnato, Laser Phys. 19, 686 (2009).

[164] J.A. Seman, E.A. Henn, M. Haque, R.F. Shiozaki, E.R. Ramos, M. Caracanhas, P. Castilho, C. Castelo Branco, P.E. Tavares, F.J. Poveda-Cuevas, G. Roati, K.M. Magalhães, and V.S. Bagnato, Phys. Rev. A 82, 033616 (2010).

[165] E.A. Henn, J.A. Seman, G. Roati, K.M. Magalhães, V.S. Bagnato, Phys. Rev. Lett. 103, 045301 (2009).

[166] R.F. Shiozaki, G.D. Telles, V.I. Yukalov, V.S. Bagnato, Laser Phys. Lett. 8, 393 (2011).

[167] J.A. Seman, E.A. Henn, R.F. Shiozaki, G. Roati, F.J. Poveda-Cuevas, K.M. Magalhães, V.I. Yukalov, M. Tsubota, M. Kobayashi, K. Kasamatsu, V.S. Bagnato, Laser Phys. Lett. 8, 691 (2011).

[168] J. Dziarmaga, Adv. Phys. 59, 1063 (2010).

[169] V.I. Yukalov, Laser Phys. Lett. 8, 485 (2011).

[170] A. Polkovnikov, K. Sengupta, A. Silva, and M. Vengalattore, Rev. Mod. Phys. 83, 863 (2011). 


\section{Figure Caption}

Fig. 1. Qualitative phase diagram showing the sequence of states on the plane of the pumping field amplitude versus pumping time. 


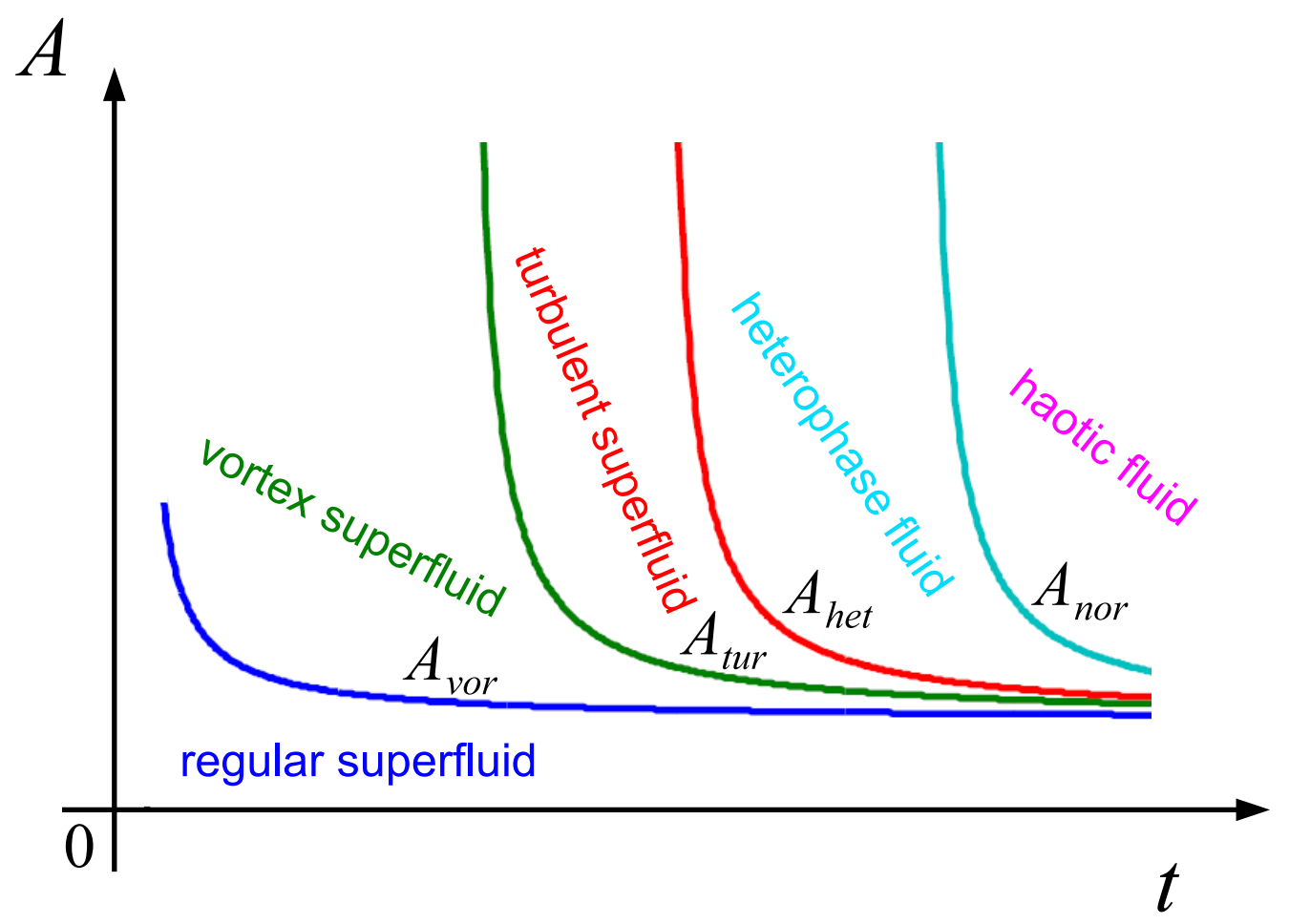

Figure 1: Qualitative phase diagram showing the sequence of states on the plane of the pumping field amplitude versus pumping time. 